Revistade
Economida
Contemporâned

\title{
VOLATILIDADE DO CÂMBIO E SEUS EFEITOS SOBRE A EXPORTAÇÃO BRASILEIRA PARA OS EUA
}

\author{
Daniel Morais de Souza $a^{a}$ \\ Fábio Júnior Clemente Gama ${ }^{b}$ \\ Júlia Goes da Silva Carmo ${ }^{c}$ \\ Cláudio Roberto Fóffano Vasconcelos ${ }^{d}$
}

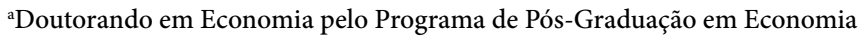
da Universidade Federal de Juiz de Fora (PPGE/UFJF). Juiz de Fora, MG, Brasil. ORCID: https://orcid.org/0000-0002-3310-6157.

${ }^{\mathrm{b}}$ Doutor em Economia pelo Programa de Pós-Graduação em Economia da Universidade Federal de Juiz de Fora (PPGE/UFJF). Juiz de Fora, MG, Brasil. ORCID: https://orcid.org/0000-0003-3772-411X.
\end{abstract}

${ }^{c}$ Doutora em Economia pelo Programa de Pós-Graduação em Economia da Universidade Federal de Juiz de Fora (PPGE/UFJF), economista da UFJF. Juiz de Fora, MG, Brasil. ORCID: https://orcid.org/0000-0002-0187-2447.

\footnotetext{
d Professor Permanente do Programa de Pós-Graduação em Economia da Universidade Federal de Juiz de Fora (PPGE/UFJF). Juiz de Fora, MG, Brasil. ORCID: https://orcid.org/0000-0003-4947-9895.
}

Recebido em 31 outubro 2019

Aceito em 09 fevereiro 2021

\begin{abstract}
RESUMO: Este trabalho investigou a influência da volatilidade cambial sobre as exportações brasileiras para os Estados Unidos entre janeiro de 1999 a fevereiro de 2017. Para tanto, empregou-se o teste de fronteira de Pesaran em uma estrutura ARDL. Adicionalmente foram construídas medidas não lineares para a volatilidade cambial a fim de verificar se choques positivos e negativos no câmbio afetam igualmente sua volatilidade. As medidas não lineares propostas indicaram que choques positivos no câmbio implicam em maior volatilidade para os períodos seguintes. Os resultados dos testes de Pesaran mostraram que a influência a longo prazo da volatilidade cambial nas exportações é similar para as diferentes medidas, contudo, os modelos considerando medidas não lineares obtiveram mais relações positivas do que os com medidas lineares. Os setores negativamente afetados foram produtos com

Correspondência para: Daniel Morais de Souza

Contato:dmorais@ice.ufjf.br
\end{abstract}


dependência do capital externo e produtos manufaturados ou com baixo valor agregado. Os setores positivamente afetados foram produtos sem dependência do capital externo ou com demanda altamente elástica.

PALAVRAS-CHAVE: volatilidade cambial; exportações; teste de fronteira de Pesaran. CLASSIFICAÇÃO JEL: F31; F41; C53. 


\title{
EXCHANGE RATE VOLATILITY AND ITS EFFECTS ON BRAZILIAN EXPORTS TO THE US
}

\begin{abstract}
This paper investigated the influence of exchange-rate volatility on Brazilian exports to the US from January 1999 to February 2017. For this purpose, we used Pesaran's bound test in an ARDL framework. Moreover, we built nonlinear measures for exchange-rate volatility to verify whether positive and negative exchange rate shocks affect equally on volatility. The proposed nonlinear measures indicated that positive exchange rate shocks imply greater volatility in the following periods. The results showed that long-term exchange rate volatility affected the exports similarly for the different measures. However, we found more positive relationships in the models using nonlinear measures than in those using linear ones. The sectors negatively affected were external capital-dependent products and manufactured or low value-added products. In contrast, the ones positively affected were products with no dependence on external capital or products with highly elastic demand.
\end{abstract}

KEYWORDS: exchange rate volatility; exportations; Pesaran's bound testing. 


\section{INTRODUÇÃO}

A volatilidade da taxa de câmbio é variável relevante para o comércio internacional tendo em vista as incertezas inerentes que afetam a decisão dos exportadores e importadores. Na literatura econômica o tema tornou-se ainda mais abordado após o encerramento do sistema Bretton Woods em 1971, quando boa parte das nações passaram a adotar o câmbio flutuante ou parcialmente controlado. Diante da tendência, surgiu a necessidade de conhecer os efeitos do novo sistema sobre diversas variáveis econômicas, entre elas, o comércio internacional. Desde então, diversos estudos foram desenvolvidos na tentativa de esclarecer o efeito da taxa de câmbio e de sua volatilidade sobre o volume de comércio internacional.

Não obstante, os resultados dos trabalhos aplicados para verificar os efeitos da volatilidade do câmbio sobre o fluxo de comércio internacional não são unânimes quanto à direção e significância da relação, como expõem Bahmani-Oskooee e Hegerty (2007). A incerteza sobre o câmbio pode gerar tanto aumento quanto redução no fluxo de comércio, dependendo dos países envolvidos, da mensuração da volatilidade, bem como da amostra selecionada. Deste modo, não é possível estabelecer previamente qual o impacto da volatilidade ao fluxo de comércio, sendo necessárias análises empíricas.

Segundo Hooper e Kohlhagen (1978) e Côté (1994), a hipótese de que a volatilidade reduz o volume de comércio é fundamentada na suposição de que a dúvida em torno das receitas, o insuficiente desenvolvimento do mercado e a aversão ao risco podem afetar o exportador reduzindo o volume de bens enviados ao exterior. A relação negativa entre a volatilidade do câmbio com o fluxo de comércio entre diferentes países é resultado de estudos como os de Esquivel e Larraín (2002), Brodsky, (1984), Hooper e Kohlhagen (1978), Grier e Smallwood (2007), Kafle (2011), Verheyen (2012), Héricourt e Poncet (2013), Kim (2017) e Aftab, Syed e Katper (2017).

Por outro lado, há autores que encontram evidências de que a volatilidade do câmbio possa ter associação positiva com o fluxo de comércio. Segundo De Grauwe (1988), se o efeito-renda sobressai ao efeito-substituição, comércio e volatilidade do câmbio se relacionariam positivamente. $\mathrm{O}$ autor argumenta que se os exportadores são pouco avessos ao risco, uma maior variabilidade do câmbio pode aumentar a utilidade marginal esperada da receita de exportação, refletindo em maior oferta de produtos exportáveis. Giovannini (1988) e Klein (1990) acrescentam que, se as firmas são neutras ao risco, a queda da demanda frente a elevação da volatilidade do câmbio pode ser contrabalanceada pela queda nos preços e pela demanda inelástica, incorrendo em um aumento do valor exportado. Corroborando esta hipótese, De Grauwe (1988), Klein (1990) e Asseery e Peel (1991) encontraram associação positiva entre a volatilidade do câmbio e o fluxo de comércio. 
Por fim, estudos apontam a possibilidade de a volatilidade do câmbio não afetar significativamente o fluxo de comércio, como visto em Bailey, Tavlas e Ulan (1987), Medhora (1990) e De Vita e Abbott (2004). Nesse âmbito da discussão teórica, há autores que defendem que o efeito da volatilidade do câmbio sobre o comércio internacional seja indeterminado, como conclui McKenzie (1999), devido ao sunk cost, que mantém a firma no mercado global, bem como a existência e o nível de desenvolvimento de mercado futuro, que podem assegurar os preços de venda e reduzir a incerteza dos ganhos de exportação.

Em termos de literatura nacional, também não há consenso sobre a associação entre a volatilidade cambial e o fluxo de comércio. Coes (1981) analisou o efeito da volatilidade do câmbio em setores desagregados da economia brasileira entre 1957 e 1974. O autor concluiu que bens do setor agrícola e manufaturados podem ser afetados de forma diferente pela incerteza da taxa de câmbio, sendo que, para manufaturados a relação é, em geral, significativa e positiva, enquanto é negativa para setores de bebidas e produtos da borracha.

Gonzaga e Terra (1997) analisaram a influência dos planos de estabilização e das oscilações da inflação sobre a volatilidade da taxa real de câmbio bem como a estimação da função de oferta do exportador para o Brasil. As medidas para a volatilidade da taxa real de câmbio utilizadas foram obtidas pelo desvio padrão móvel das taxas reais de câmbio entre 1980 e 1995. Os autores consideram exportações compostas majoritariamente por produtos com alto grau de competitividade no exterior, pressupondo exportadores avessos ao risco em um ambiente competitivo, o que caracteriza as economias em desenvolvimento. Os resultados indicam que o coeficiente de volatilidade é negativo, a despeito de parte da relação estimada não ser significativa.

Com desagregação para cinco setores, compreendendo amostra de 64 produtos, Bittencourt, Larson e Thompson (2007) analisaram os efeitos da volatilidade da taxa real de câmbio entre países do Mercosul sobre comércio no período de 1989 a 2002. Para cálculo da volatilidade os autores utilizaram as medidas do desvio padrão móvel e da volatilidade de Peree e Steinherr, sendo que ambas levaram a resultados similares. Os autores, com uso de equação gravitacional, concluíram que o aumento da volatilidade reduz o fluxo de comércio bilateral para o caso brasileiro, em consonância com o obtido por Gonzaga e Terra (1997), mas acrescentam que o mesmo efeito negativo é observado com a variabilidade das taxas de seus parceiros do Mercosul. Os setores de mineração e óleo, e de manufaturados se mostraram os mais sensíveis à volatilidade da taxa real de câmbio.

Outros trabalhos também utilizaram o modelo gravitacional para verificar os efeitos da volatilidade da taxa real de câmbio brasileira no comércio internacional, como Silva, Freitas e Mattos (2016) e Bittencourt e Campos (2014). Os últimos autores investigaram 
os impactos da instabilidade da taxa de câmbio e outras variáveis sobre o fluxo de comércio para nove países da América do Sul, incluindo o Brasil. Os resultados indicam que quanto maior a instabilidade menor o volume de comércio entre os países, corroborando o efeito negativo de outros trabalhos. Entre as justificativas para tal comportamento, os autores apontam o maior risco e incerteza que desincentiva as atividades do comércio externo, bem como a tardia capacidade das empresas exportadoras e importadoras alterarem, no curto prazo, seus fatores de produção.

Silva, Freitas e Mattos (2016) corroboraram os resultados do estudo de Bittencourt e Campos (2014) ao avaliarem, no período de 1989 a 2011, o efeito da volatilidade cambial brasileira nos fluxos de exportações e importações de cinco setores com seus principais parceiros, quais sejam, China, Estados Unidos (EUA), Argentina, Holanda, Japão, Alemanha e Chile. Os autores concluem que os fluxos de comércio setorial entre o Brasil e esses parceiros comerciais foram negativamente afetados por incertezas em sua taxa de câmbio. Assim, apontam para a necessidade de manter a estabilidade econômica, reduzindo as incertezas relativas ao movimento cambial para, no caso brasileiro, buscar mais elevados fluxos comerciais bilaterais.

Para o período de 1973 a 2004, Arize, Osang e Slottje (2008) investigaram os impactos da incerteza cambial sobre os fluxos de exportação de oito países da América Latina, incluindo o Brasil. Os autores encontraram uma relação de longo prazo entre a volatilidade da taxa real de câmbio efetiva e a demanda por exportação daqueles países, sendo esta negativa e significativa. Nesse estudo, os autores aplicam técnicas de cointegração para avaliar tais efeitos sobre o volume exportado pelos países.

Utilizando a análise de cointegração pela abordagem Autoregressive Distributed Lag (ARDL), Bahmani-Oskooee, Harvey e Hegerty (2013) avaliaram o impacto da volatilidade da taxa real de câmbio brasileira sobre a relação de comércio de 123 e 103 setores exportados e importados, respectivamente, para os Estados Unidos, no período de 1971 a 2010 . Os autores utilizaram o desvio padrão para obtenção da volatilidade da taxa real, e concluem que, do total, 29 e 24 setores de exportação e importação, nesta ordem, têm significativos coeficientes de volatilidade. Ademais, os coeficientes de sinal positivo superam os negativos, na proporção de dois para um, como expressam os autores. Os resultados apontam que as exportações agrícolas brasileiras são prejudicadas, ao passo que as importações de máquinas dos Estados Unidos não são afetadas. Por fim, os autores afirmam que os produtos de baixa participação no comércio internacional respondem mais ao aumento da incerteza se comparados àqueles caracterizados como exportadores.

Estimando modelos ARDL, Aguirre, Ferreira e Notini (2007) encontraram evidências de que a volatilidade cambial afeta negativamente as exportações brasileiras de manufaturados para o período de 1986 a 2002. Como proxy de volatilidade, os autores calcularam o desvio padrão móvel da taxa de câmbio, que corrobora a relação negativa 
da volatilidade com as exportações, e o método GARCH, que, no entanto, levou a coeficientes não significativos para tal relação. Os autores apontam como explicação para a discordância dos resultados que os efeitos de clustering e forward looking decorrentes do modelo GARCH seriam menos importantes no caso brasileiro.

Corrêa, Vasconcelos e Lima Junior (2018), também por meio do mesmo método de análise de cointegração, mostraram que o fluxo de comércio brasileiro para o Mercosul é negativamente afetado pela volatilidade do câmbio no Brasil, enquanto para Estados Unidos e União Europeia, a relação não é tão clara. Os autores utilizaram o desvio padrão para apurar a volatilidade do câmbio, e dados para as exportações de produtos básicos, semimanufaturados e manufaturados, de janeiro de 2000 a outubro de 2014 .

A divergência quanto ao sinal da relação da volatilidade do câmbio com o volume de exportações sugere a necessidade de mais pesquisas relativas ao tema para melhor compreensão das características de mercado de cada setor. Investigar quais seriam o sentido e a magnitude do efeito de flutuações na taxa de câmbio em setores específicos de importação e exportação podem dar aos agentes econômicos um nível maior de certeza em relação ao cenário do comércio internacional e, consequentemente, maiores ganhos para a economia. Ademais, outras lacunas podem ser preenchidas, como a inserção de novas medidas de volatilidade nos modelos para verificar se os resultados são semelhantes. Brodsky (1984) afirma que o uso de medidas de variabilidade inapropriadas para o cálculo da volatilidade da taxa do câmbio pode produzir resultados conflitantes. ${ }^{1}$

Neste mote de pesquisa, o objetivo deste artigo é analisar e comparar as relações de longo prazo entre diferentes medidas para a volatilidade da taxa de câmbio real/dólar e o fluxo comercial Brasil/EUA. Especificamente, analisa-se o impacto da volatilidade cambial no fluxo de exportações dos cem setores mais relevantes da pauta das exportações para os EUA, desagregados em quatro dígitos da Nomenclatura Comum do Mercosul (NCM). Para este fim, foi utilizado o método de cointegração de Pesaran, Shin e Smith (2001) via modelos autorregressivos de defasagem distribuída (ARDL), o qual permite a cointegração entre variáveis $\mathrm{I}(0)$ e I(1). Além disso, construiu-se quatro medidas de volatilidade do câmbio: duas lineares (desvio padrão móvel de seis meses e GARCH) e duas não lineares (TGARCH e EGARCH), sendo as últimas duas medidas que ponderam diferentemente choques positivos e negativos.

Pode-se concluir que as diferentes medidas de volatilidade cambial produzem efeitos a longo prazo semelhantes no fluxo de comércio, evidenciando a robustez dos resultados.

\footnotetext{
1 Mais detalhes sobre medidas de volatilidade do câmbio podem ser obtidos em Kenen e Rodrik (1986) e Griebeler (2014).
} 
Em geral, a volatilidade do câmbio afeta negativamente setores com elevada dependência do capital externo e de produtos essencialmente manufaturados com baixo valor, enquanto os setores positivamente afetados foram produtos com pouca dependência do capital externo ou com demanda altamente elástica.

A próxima seção apresenta os aspectos metodológicos, ao passo que a segunda seção expõe a base de dados. Por fim, os resultados estão expostos na terceira seção, enquanto a seção seguinte traz as considerações finais.

\section{ASPECTOS METODOLÓGICOS}

O modelo de comércio proposto segue as especificações para substitutos perfeitos discutidas em Goldstein e Khan (1985). Tomando seus argumentos para o uso de tal abordagem, a equação da demanda por exportações brasileiras segue:

$$
\ln X_{t}=\alpha+\beta \ln Y_{t}+\delta \ln P_{t}+\theta \ln V o l_{t}+\varepsilon_{t}
$$

Em (1), $X_{t}$ representa o volume em dólares de exportação, $Y_{t}$ a renda externa, representada pela proxy do Produto Interno Bruto (PIB) do parceiro comercial, ${ }^{2} P_{t}$ a taxa de câmbio, $V_{o} l_{t}$ a volatilidade do câmbio, $\varepsilon_{t}$ o termo de erro e $\alpha, \beta, \delta, \theta$ os parâmetros a serem estimados.

Em termos de sinais, espera-se que a renda externa e a taxa de câmbio ( $\mathrm{R} \$ / \mathrm{U} \$$ ) afetem positivamente o fluxo de exportações. Já para o coeficiente $\delta$, assume-se a hipótese de que um aumento na taxa de câmbio amplia o volume exportado, pois, a elevação do câmbio torna os produtos nacionais mais baratos em comparação aos internacionais similares. Por fim, para o coeficiente que relaciona volume exportado e volatilidade há a discussão do sinal de $\theta$, podendo este ser negativo ou positivo.

Na literatura especializada, diversos trabalhos utilizam o teste de fronteira de Pesaran em estrutura ARDL para investigar a influência da volatilidade do câmbio sobre o volume das exportações (SHAIKH; HONGBING, 2015). A metodologia vai ao encontro desta investigação pois o teste é uma técnica de cointegração que, ao contrário das técnicas comumente utilizadas - Engle e Granger (1987) e Johansen (1988, 1991) - não exige que as variáveis sejam integradas de mesma ordem, permitindo que séries $\mathrm{I}(0) \mathrm{e}$ I(1) se relacionem em equação única de longo prazo.

\footnotetext{
2 Como não foram encontrados dados mensais do PIB norte-americano, utilizou-se seu índice de produção industrial.
} 
O teste de Pesaran desenvolvido na estrutura ARDL consiste em verificar se existe cointegração entre um grupo de variáveis, construindo regressões estimadas por mínimos quadrados com defasagens da variável dependente e das variáveis explicativas. Para verificar a existência desta relação de longo prazo dentro da estrutura ARDL, o primeiro passo é estimar o seguinte modelo de correção de erros irrestrito por mínimos quadrados ordinários (MQO):

$$
\begin{aligned}
& \Delta \ln X_{t}=a_{0}+\sum_{i=1}^{p} a_{1 i} \Delta \ln X_{t-i}+\sum_{i=1}^{p} a_{2 i} \Delta \ln Y_{t-i}+\sum_{i=1}^{p} a_{3 i} \Delta \ln P_{t-i}+ \\
& \sum_{i=1}^{p} a_{4 i} \Delta \ln V o l_{t-i}+b_{1} \ln X_{t-1}+b_{2} \ln Y_{t-1}+b_{3} \ln P_{t-1}+b_{4} \ln V o l_{t-1}+\varepsilon_{t}
\end{aligned}
$$

Estimado o modelo, testa-se a hipótese conjunta de que os multiplicadores de longo prazo das variáveis defasadas de (2) são todos iguais a zero, contra a alternativa de que pelo menos um seja diferente de zero. É importante salientar que a estatística $F$ obtida pelo teste de Wald possui uma distribuição não padronizada, sendo necessário a tabela dos valores limites críticos. ${ }^{3}$ Se a estatística de teste for menor que o valor crítico inferior, não há evidência da existência de cointegração; se estiver entre os valores críticos, o teste se torna inconclusivo; e, se a estatística for maior que o valor crítico superior, decide-se pela existência de cointegração. No caso de teste inconclusivo, averígua-se a existência de cointegração pela abordagem de Banerjee, Dolado e Mestre (1998), que considera a estatística $t$ da estimativa do mecanismo de correção de erros (ECM). Esta estatística é comparada com o valor crítico estabelecido em Banerjee, Dolado e Mestre (1998) considerando o tamanho da amostra e o número de regressores.

Verificada a existência de cointegração, deve-se selecionar a melhor especificação do ARDL segundo algum critério. A representação do modelo é denotada por ARDL $\left(p, q_{1}, \ldots, q_{k}\right)$, onde $p$ é o número de defasagens da variável dependente e $q_{k}$ é o número de defasagens da $k$-ésima variável explicativa. O modelo $\operatorname{ARDL}\left(p, q_{1}, \ldots, q_{k}\right)$ aplicado para o contexto deste trabalho é dado por:

$$
\begin{aligned}
& \ln X_{t}=c_{0}+\sum_{i=1}^{p} c_{1 i} \ln X_{t-i}+\sum_{i=1}^{q_{1}} c_{2 i} \ln Y_{t-i}+\sum_{i=1}^{q_{2}} c_{3 i} \ln P_{t-i} \\
& +\sum_{=1}^{q_{3}} c_{4 i} \ln V \operatorname{lol}_{t-i}+u_{t}
\end{aligned}
$$

Caso seja constatada a presença de cointegração é possível transformar o modelo ARDL em uma representação de longo e curto prazo como funções não lineares dos coeficientes de (3). Para isso, igualam-se todas as variáveis de (3) às suas versões

\footnotetext{
3 Valores críticos assintóticos são fornecidos no trabalho de Pesaran, Shin e Smith (2001)..
} 
contemporâneas, e manipula-se algebricamente de modo a se obter a equação cointegrante, representada por:

$$
\ln X_{t}=d_{0}+d_{2} \ln Y_{t}+d_{3} \ln P_{t}+d_{4} \ln V o l_{t}+v_{t}
$$

$\operatorname{Em}(4), d_{0}=\frac{c_{0}}{1-\sum_{i=1}^{p} c_{1 i}}, \quad d_{m}=\frac{\sum_{i=0}^{q_{m}} c_{m i}}{1-\sum_{i=1}^{p} c_{1 i}}$ para $m=2,3,4$ e $v_{t}=\frac{u_{t}}{1-\sum_{i=1}^{p} c_{1 i}}$.

O último passo envolve estimar por MQO os coeficientes de curto prazo, como segue:

$$
\begin{aligned}
& \Delta \ln X_{t}=h_{0}+\sum_{i=1}^{p} h_{1 i} \Delta \ln X_{t-i}+\sum_{i=0}^{q_{1}} h_{2 i} \Delta \ln T_{t-i}+\sum_{i=0}^{q_{2}} h_{3 i} \Delta \ln Y_{t-i}+ \\
& \sum_{=0}^{q_{3}} h_{4 i} \Delta \ln P e_{t-i}+h_{5} \hat{v}_{t-1}+w_{t}
\end{aligned}
$$

Em (5), o termo corresponde ao mecanismo de correção de erro representado por (4) e $w_{t}$ é o termo de erro independente e identicamente distribuído seguindo uma distribuição normal com média zero e variância $\sigma^{2}$.

\section{BASE DE DADOS}

A base de dados utilizada nas estimações compreende a taxa de câmbio real ${ }^{4}$, a produção industrial dos EUA como proxy da atividade econômica e os cem principais setores das exportações brasileiras para os EUA. O período da análise é de janeiro/1999 a fevereiro/2017. O Quadro 1 apresenta as variáveis utilizadas, a unidade da variável e a fonte dos dados.

\section{Quadro 1 - Composição da base de dados e suas fontes}

\begin{tabular}{ccc}
\hline Descrição & Observação & Fonte \\
\hline $\begin{array}{c}\text { Volume de exportações } \\
\text { do Brasil para os EUA }\end{array}$ & Dólares correntes dos EUA, FOB & ALICEWEB/COMEXSTAT (2017) \\
\hline Índice de produção industrial & $\begin{array}{c}\text { Proxy: renda externa } \\
\text { dólares correntes }\end{array}$ & $\begin{array}{c}\text { BOARD OF GOVERNORS OF } \\
\text { THE FED-ERAL RESERVE } \\
\text { SYSTEM (2017) }\end{array}$ \\
\hline Taxa de câmbio real bilateral & real/dólar & INSTITUTO DE PESQUISA \\
& & ECONÔMICA APLICADA \\
(IPEA, 2017)
\end{tabular}

Fonte: Elaboração própria.

4 A taxa de câmbio real entre Brasil e EUA está definida como: $\mathrm{E}=\mathrm{eP} \mathrm{P}^{\star} \mathrm{P}$; em que, $E$ é a taxa de câmbio real, $e$ é a taxa de câmbio nominal (R\$/U\$), $P$ representa o índice de preço no Brasil e $P^{\star}$ representa o índice de preço americano. 
As séries das exportações brasileiras e do índice de produção industrial americano foram dessazonalidas ${ }^{5}$ e deflacionadas com o índice de preços ao consumidor (IPC) dos EUA. A desagregação do volume de exportações foi feita com base nos setores a quatro dígitos da NCM, sendo utilizados os cem setores mais representativos das exportações Brasil/EUA para o período em análise. Contudo, dez setores continham extensos períodos de dados faltantes, impossibilitando sua análise. Outros dez setores apresentaram dados faltantes no começo do período amostral, sendo necessário o encurtamento do período deles. Para trinta setores foi aplicado o método de interpolação Spline Cúbica ${ }^{6}$ para aproximar valores esporadicamente faltantes entre as observações.

A partir da série de câmbio construiu-se quatro medidas para a volatilidade: uma baseada no método de desvio padrão móvel considerando um intervalo de tempo de seis meses; e outras três baseadas em modelos que abordam a heterocedasticidade condicional (GARCH, TGARCH E EGARCH). O método do desvio padrão móvel para seis meses da taxa de câmbio é:

$$
\sigma_{t}=\left[\frac{\sum_{i=1}^{6}\left(P_{t+i-1}-P_{t+i-2}\right)^{2}}{6}\right]^{\frac{1}{2}}
$$

Em (6), $\sigma_{t}$ representa o desvio padrão móvel para seis meses no tempo $t$. O indicador do desvio padrão é frequentemente utilizado como medida de variabilidade com o intuito de minimizar os efeitos espúrios gerados por períodos isolados em que se observa elevada volatilidade, isto é, o indicador ameniza problemas de outliers na construção da medida.

Não obstante, as séries econômicas podem apresentar características que violam os pressupostos da análise de regressão padrão. De acordo com Bollerslev, Chou e Kroner (1992), as séries financeiras são tipicamente heterocedásticas e leptocúrticas, sendo necessário modelar a volatilidade como uma série temporal condicionada ao comportamento passado. Assim, desde sua introdução por Engle (1982), o método de heterocedasticidade condicional ARCH e suas generalizações são aplicados à análise da volatilidade cambial por permitirem a modelagem temporal das variâncias. Segundo Bueno (2011), a principal contribuição desses modelos é a distinção de uso entre momentos de segunda ordem condicionais e não condicionais, o que permite captar a dinâmica do componente de incerteza sobre os movimentos das séries econômicas. Além disso, generalizações do modelo $\mathrm{ARCH}$, tais como os modelos EGARCH e

\footnotetext{
5 As séries foram dessazonalizadas pelo filtro Census X-13..

6 O método de interpolação Spline Cúbica permite aproximar valores inexistentes da série através de um polinômio cúbico.
} 
TGARCH, permitem que choques positivos e negativos possam afetar a medida de volatilidade com pesos diferentes.

Para o método das heterocedasticidades condicionais da taxa de câmbio, (7) representa a equação para a média da taxa de câmbio de todos os modelos, e as equações (8), (9), (10) representam os modelos $\operatorname{GARCH}(1,1)$, TGARCH(1,1) e $\operatorname{EGARCH}(1,1)$, de acordo com Brooks (2008), respectivamente:

$$
\begin{gathered}
\Delta P_{t}=\tau_{0}+\tau_{1} \Delta P_{t-1}+\tau_{2} \Delta P_{t-2}+\varepsilon_{t} \\
\sigma_{t}^{2}=\alpha_{0}+\alpha_{1} \varepsilon_{t-1}^{2}+\beta_{1} \sigma_{t-1}^{2} \\
\sigma_{t}^{2}=\alpha_{0}+\alpha_{1} \varepsilon_{t-1}^{2}+\beta_{1} \sigma_{t-1}^{2}+\alpha_{2} \varepsilon_{t-1}^{2} I_{t-1} \\
\ln \ln \left(\sigma_{t}^{2}\right)=\alpha_{0}+\beta_{2} \ln \ln \left(\sigma_{t-1}^{2}\right)+\alpha_{3}\left|\frac{\varepsilon_{t-1}}{\sigma_{t-1}}\right|+\alpha_{4} \frac{\varepsilon_{t-1}}{\sigma_{t-1}}
\end{gathered}
$$

Nesse conjunto de equações, $\Delta$ representa a primeira diferença, $\varepsilon_{t}$ o erro heterocedástico aleatório ; $\sigma_{\mathrm{t}}^{2}$ a variância condicional no período $t$; $I_{t-1}$ é variável dummy que assume 1 se $\varepsilon_{t-1}<0$ e 0 caso contrário. $\tau_{0}, \tau_{1}, \tau_{2}, \alpha_{0}, \alpha_{1}, \alpha_{2}, \alpha_{3}, \alpha_{4}, \beta_{1}$ e $\beta_{2}$ são parâmetros a estimar, sendo que $\alpha_{2}$ e $\alpha_{3}$ representam os componentes de assimetria dos modelos TGARCH $(1,1)$ e EGARCH $(1,1)$, respectivamente.

\section{RESULTADOS}

A Figura 1 apresenta a dinâmica das medidas construídas para a volatilidade do câmbio real/dólar. Em geral, a volatilidade não foi elevada, contudo sofreu choques pontuais em momentos de instabilidades. Como exemplo, em 1999 o efeito da crise russa ainda perdurava sobre as economias emergentes, quando fuga de capitais, aliada a implementação do regime de câmbio flutuante, propiciou forte elevação da taxa de câmbio. Outro pico de volatilidade aconteceu entre 2002 e 2003. A incerteza no mercado diante da eleição presidencial, associada aos reflexos negativos da bolha financeira do início dos anos 2000, fez com que os agentes optassem por ativos mais seguros, implicando em flutuação dos recursos externos em dólar no país. O mesmo acontece nos períodos entre 2008 e 2009 - devido à crise do subprime - e 2014 e 2017 - em face da crise política. 
Figura 1 - Volatilidades da taxa de câmbio

DESVIO PADRÃO MÓVEL 6
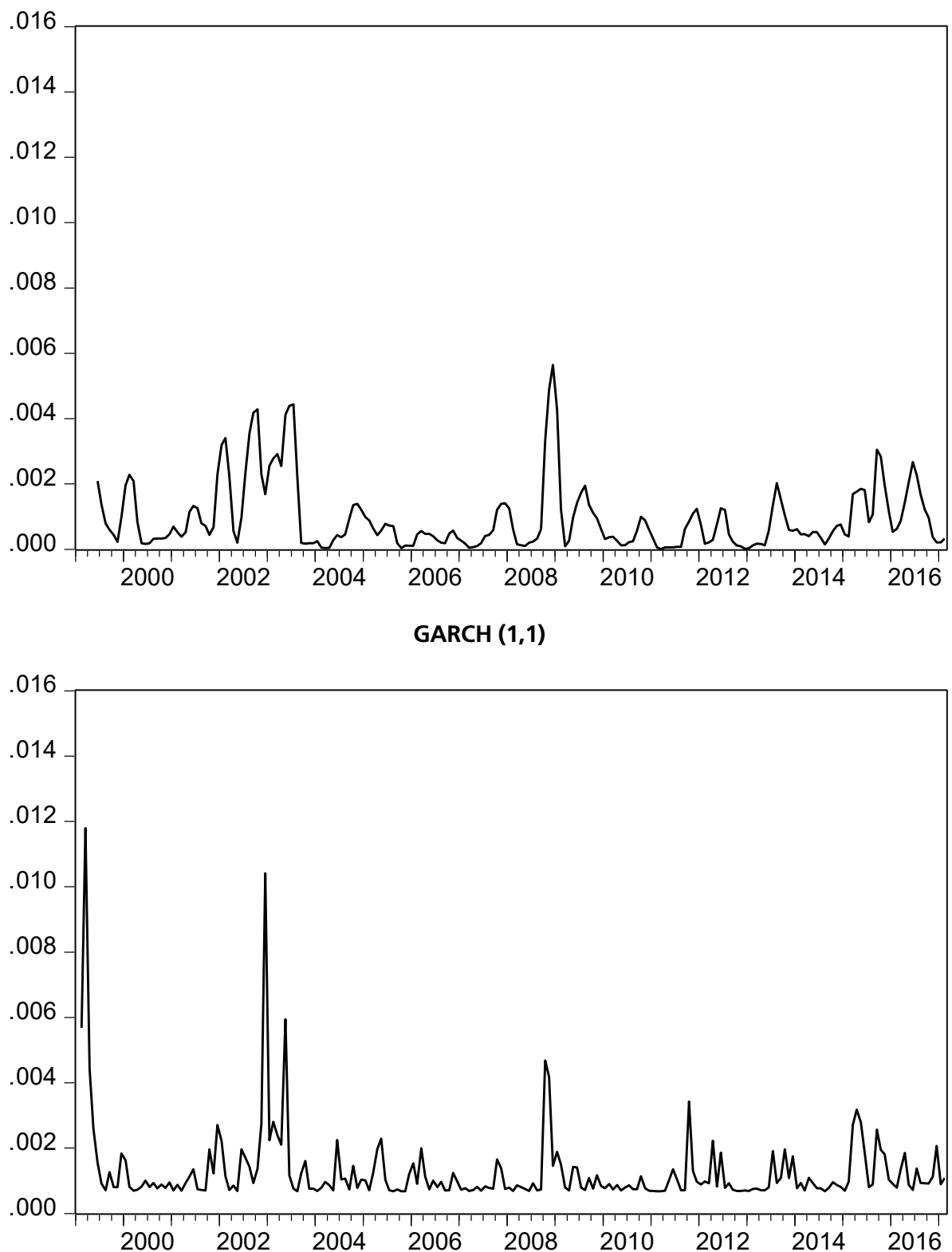
$\operatorname{EGARCH}(1,1)$
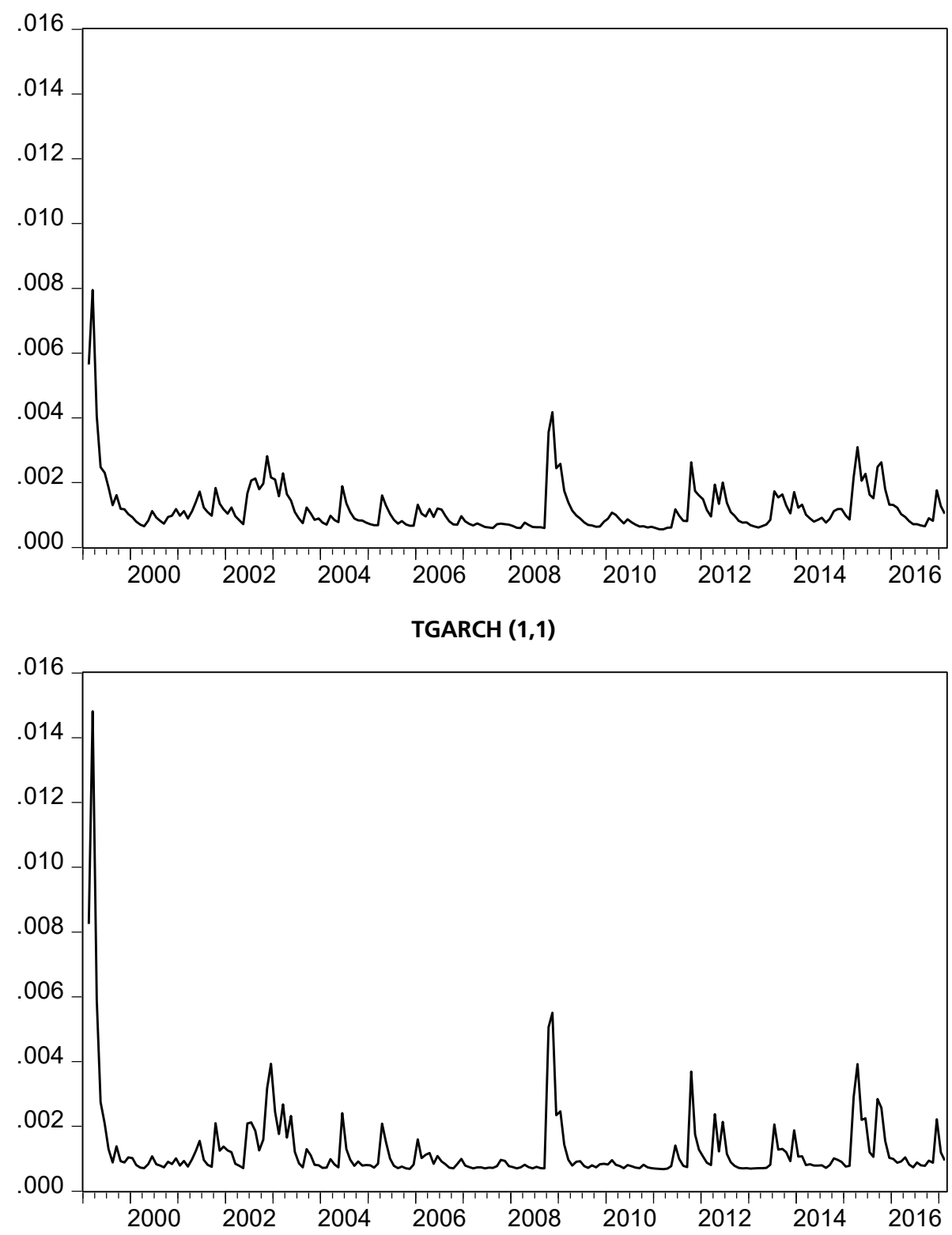

Fonte: Elaboração própria. 


\subsection{POLÍTICA DE ACUMULAÇÃO DE RESERVAS INTERNACIONAIS}

Um ponto importante a se destacar sobre as medidas de volatilidade TGARCH e EGARCH são as estimativas dos componentes assimétricos. Para o caso do modelo TGARCH(1,1), $\alpha_{2}$ foi significativo e apresentou sinal negativo, indicando que os choques positivos na taxa de câmbio implicam em maior volatilidade para os períodos seguintes. De forma similar, o parâmetro $\alpha_{3}$ do modelo $\operatorname{EGARCH}(1,1)$ foi significativo e apresentou sinal positivo, corroborando o modelo TGARCH(1,1). A Tabela 1 traz as estatísticas dos modelos $\operatorname{GARCH}(1,1)$, TGARCH(1,1) e $\operatorname{EGARCH}(1,1)$.

\section{Tabela 1 - Estatísticas dos modelos de heterocedasticidades condicionais para a volatilidade da taxa de câmbio}

\begin{tabular}{cccc}
\hline Parâmetros & GARCH(1,1) & TGARCH(1,1) & EGARCH(1,1) \\
\hline Média & & & $-0,002$ \\
$\widehat{\tau_{0}}$ & $-0,002$ & $-0,001$ & $0,316^{*}$ \\
$\widehat{\tau_{1}}$ & $0,338^{*}$ & $0.149^{*}$ & $-0,152^{*}$ \\
\hline Variância & $-0,154^{*}$ & & $-1,989^{*}$ \\
$\widehat{\tau_{0}}$ & & & - \\
$\widehat{\alpha_{1}}$ & $0,001^{*}$ & $0,001^{*}$ & - \\
$\widehat{\alpha_{2}}$ & $0,405^{*}$ & $0,448^{*}$ & $0,263^{*}$ \\
$\widehat{\alpha_{3}}$ & - & $-0,343^{* *}$ & $0,192^{*}$ \\
$\widehat{\alpha_{4}}$ & - & - & - \\
$\widehat{\beta_{1}}$ & - & - & $0,741^{*}$ \\
\hline$\beta_{2}$ & 0,092 & $0,320^{*}$ & - \\
\hline
\end{tabular}

Nota: $\left(^{*}\right)$ e $\left(^{* *}\right)$ valores significativos a um nível de $5 \%$ e10\%, respectivamente.

Fonte: Elaboração própria.

Os resultados indicam que as depreciações cambiais têm papel mais relevante para determinar o grau de incerteza dos agentes em relação aos movimentos do câmbio quando comparadas às apreciações cambiais. $\mathrm{O}$ fato do Brasil ser uma economia exportadora de commodities, e de relativo risco aos investimentos externos, pode explicar a maior incerteza gerada pelas variações positivas na taxa de câmbio. Dado que o mercado de commodities tem forte competição entre os países, choques positivos na taxa de câmbio implicariam uma rápida elevação das exportações.

Por outro lado, o considerável risco de perdas para os investimentos externos faz com que os choques positivos impliquem em fugas de recursos. Assim, após choque positivo, observam-se dois movimentos da taxa de câmbio: i) depreciação perante a fuga de capitais por meio da conta de capitais; e, ii) elevação diante da entrada de recursos 
via balança comercial. Essa variação bidirecional implica em alta volatilidade do câmbio, em contrapartida, choques negativos no câmbio acarretam, de forma mais imediata, queda das exportações. Isso se deve aos riscos associados às economias subdesenvolvidas fazerem com que a conta capital seja mais rígida às valorizações cambiais, implicando em movimento mais acentuado em uma direção.

Para atender a metodologia de cointegração de Pesaran, foi verificada a ordem de integração por meio dos testes de raiz unitária Augmented Dickey-Fuller (ADF) e Phillips-Perron (PP). Ambos concluem que todas as séries possuem ordem de integração menor do que dois. A taxa de câmbio real se mostrou I(1), enquanto as exportações brasileiras são em maioria I(0). A série de produção industrial dos EUA é I(1), ao passo que as séries de volatilidade são I(0). Como todas as séries são I(0) ou I(1), a metodologia de cointegração de Pesaran é adequada para o interesse do estudo, cujos resultados estão na Tabela I do Apêndice.

Pode-se observar que 37 setores apresentaram cointegração com todas as medidas de volatilidade, ao passo que 51, em pelo menos uma das medidas de volatilidade. A Tabela 2 traz os coeficientes das volatilidades do câmbio encontrados nas relações de cointegração, que corresponde a influência das volatilidades do câmbio nas exportações.

Tabela 2 - Resultados dos coeficientes das volatilidades na relação de cointegração

\begin{tabular}{|c|c|c|c|c|c|c|c|c|c|c|c|c|}
\hline \multirow[b]{2}{*}{ Setores } & \multicolumn{3}{|c|}{ Desvio Padrão móvel 6} & \multicolumn{3}{|c|}{$\operatorname{GARCH}(1,1)$} & \multicolumn{3}{|c|}{$\operatorname{TGARCH}(1,1)$} & \multicolumn{3}{|c|}{$\operatorname{EGARCH}(1,1)$} \\
\hline & Pos & Neg & $\begin{array}{c}\text { Não } \\
\text { afetado }\end{array}$ & Pos & Neg & $\begin{array}{c}\text { Não } \\
\text { afetado }\end{array}$ & Pos & Neg & $\begin{array}{c}\text { Não } \\
\text { afetado }\end{array}$ & Pos & Neg & $\begin{array}{c}\text { Não } \\
\text { afetado }\end{array}$ \\
\hline 901 & 0 & 0 & 1 & 0 & 0 & 1 & 0 & 0 & 1 & 0 & 0 & 1 \\
\hline 904 & 0 & 0 & 1 & 0 & 0 & 1 & 0 & 0 & 1 & 0 & 0 & 1 \\
\hline 1005 & 0 & 0 & 1 & 0 & 0 & 1 & - & - & - & - & - & - \\
\hline 1602 & 0 & 0 & 1 & 0 & 0 & 1 & 0 & 0 & 1 & 0 & 0 & 1 \\
\hline 1701 & 0 & $1 * * *$ & 0 & 0 & $1 * * *$ & 0 & 0 & $1^{*}$ & 0 & 0 & 0 & 1 \\
\hline 1804 & - & - & - & 0 & 0 & 1 & - & - & - & - & - & - \\
\hline 2009 & 0 & $1^{*}$ & 0 & 0 & 0 & 1 & 0 & 0 & 1 & 0 & $1^{*}$ & 0 \\
\hline 2101 & 0 & 0 & 1 & 0 & 0 & 1 & 0 & 0 & 1 & 0 & 0 & 1 \\
\hline 2207 & 0 & 0 & 1 & 0 & 0 & 1 & $1^{*}$ & 0 & 0 & 0 & 0 & 1 \\
\hline 2401 & 0 & 0 & 1 & 0 & 0 & 1 & 0 & 0 & 1 & 0 & 0 & 1 \\
\hline 2709 & - & - & - & 0 & 0 & 1 & 0 & 0 & 1 & 0 & 0 & 1 \\
\hline 2710 & 0 & 0 & 1 & 0 & 0 & 1 & 0 & 0 & 1 & $1 * *$ & 0 & 0 \\
\hline 2804 & $1 * * *$ & 0 & 0 & 0 & 0 & 1 & 0 & 0 & 1 & 0 & 0 & 1 \\
\hline 2825 & - & - & - & 0 & $1 * * *$ & 0 & $1 * * *$ & 0 & 0 & $1 *$ & 0 & 0 \\
\hline 2902 & 0 & $1^{* *}$ & 0 & - & - & - & 0 & 0 & 1 & - & - & - \\
\hline 2922 & 0 & $1 * * *$ & 0 & 0 & $1 * *$ & 0 & 0 & 0 & 1 & 0 & 0 & 1 \\
\hline 3004 & - & - & - & 0 & 0 & 1 & - & - & - & - & - & - \\
\hline 3005 & 0 & 0 & 1 & 0 & 0 & 1 & 0 & 0 & 1 & 0 & 0 & 1 \\
\hline 3301 & $1 * * *$ & 0 & 0 & $1 * * *$ & 0 & 0 & $1 * * *$ & 0 & 0 & $1 *$ & 0 & 0 \\
\hline 3503 & 0 & $1 * *$ & 0 & 0 & $1 * *$ & 0 & 0 & $1^{* *}$ & 0 & 0 & $1 * * *$ & 0 \\
\hline 4002 & 0 & 0 & 1 & 0 & 0 & 1 & $1 * *$ & 0 & 0 & $1 * *$ & 0 & 0 \\
\hline
\end{tabular}


Tabela 2 - Resultados dos coeficientes das volatilidades na relação de cointegração (CONTINUAÇÃO)

\begin{tabular}{|c|c|c|c|c|c|c|c|c|c|c|c|c|}
\hline \multirow[b]{2}{*}{ Setores } & \multicolumn{3}{|c|}{ Desvio Padrão móvel 6} & \multicolumn{3}{|c|}{$\operatorname{GARCH}(1,1)$} & \multicolumn{3}{|c|}{$\operatorname{TGARCH}(1,1)$} & \multicolumn{3}{|c|}{$\operatorname{EGARCH}(1,1)$} \\
\hline & Pos & Neg & $\begin{array}{c}\text { Não } \\
\text { afetado }\end{array}$ & Pos & Neg & $\begin{array}{c}\text { Não } \\
\text { afetado }\end{array}$ & Pos & Neg & $\begin{array}{c}\text { Não } \\
\text { afetado }\end{array}$ & Pos & Neg & $\begin{array}{c}\text { Não } \\
\text { afetado }\end{array}$ \\
\hline 4107 & 0 & 0 & 1 & 0 & 0 & 1 & 0 & $1 *$ & 0 & 0 & $1 *$ & 0 \\
\hline 4412 & - & - & - & - & - & - & - & - & - & 0 & 0 & 1 \\
\hline 4703 & $1 * * *$ & 0 & 0 & $1 * *$ & 0 & 0 & $1 * * *$ & 0 & 0 & $1 * * *$ & 0 & 0 \\
\hline 4802 & 0 & 0 & 1 & 0 & 0 & 1 & 0 & 0 & 1 & 0 & 0 & 1 \\
\hline 5607 & 0 & 0 & 1 & 0 & 0 & 1 & $1 *$ & 0 & 0 & $1 * *$ & 0 & 0 \\
\hline 6402 & 0 & 0 & 1 & 0 & 0 & 1 & 0 & 0 & 1 & 0 & 0 & 1 \\
\hline 6403 & 0 & $1^{*}$ & 0 & 0 & 0 & 1 & 0 & $1 * *$ & 0 & 0 & $1 * * *$ & 0 \\
\hline 6802 & - & - & - & - & - & - & 0 & 0 & 1 & 0 & $1^{*}$ & 0 \\
\hline 7103 & 0 & 0 & 1 & $1 *$ & 0 & 0 & $1^{*}$ & 0 & 0 & $1^{*}$ & 0 & 0 \\
\hline 7108 & - & - & - & - & - & - & - & - & - & 0 & $1 * * *$ & 0 \\
\hline 7201 & - & - & - & 0 & $1 * *$ & 0 & 0 & $1 * *$ & 0 & 0 & $1 * * *$ & 0 \\
\hline 7202 & 0 & 0 & 1 & 0 & 0 & 1 & 0 & 0 & 1 & 0 & 0 & 1 \\
\hline 7207 & 0 & 0 & 1 & 0 & 0 & 1 & $1^{*}$ & 0 & 0 & $1^{*}$ & 0 & 0 \\
\hline 7213 & 0 & 0 & 1 & 0 & 0 & 1 & 0 & 0 & 1 & 0 & 0 & 1 \\
\hline 7219 & - & - & - & 0 & 0 & 1 & 0 & 0 & 1 & 0 & 0 & 1 \\
\hline 7224 & 0 & 0 & 1 & 0 & 0 & 1 & 0 & 0 & 1 & 0 & 0 & 1 \\
\hline 7228 & 0 & 0 & 1 & 0 & 0 & 1 & 0 & 0 & 1 & 0 & 0 & 1 \\
\hline 7304 & 0 & 0 & 1 & 0 & 0 & 1 & 0 & 0 & 1 & 0 & 0 & 1 \\
\hline 8112 & 0 & $1^{* *}$ & 0 & 0 & $1^{*}$ & 0 & 0 & $1^{*}$ & 0 & 0 & $1 * *$ & 0 \\
\hline 8207 & 0 & 0 & 1 & 0 & 0 & 1 & 0 & 0 & 1 & 0 & 0 & 1 \\
\hline 8409 & - & - & - & - & - & - & 0 & $1^{*}$ & 0 & 0 & $1 * *$ & 0 \\
\hline 8411 & 0 & 0 & 1 & 0 & 0 & 1 & 0 & 0 & 1 & 0 & 0 & 1 \\
\hline 8412 & 0 & 0 & 1 & 0 & 0 & 1 & - & - & - & - & - & - \\
\hline 8429 & 0 & $1 * * *$ & 0 & 0 & $1 * * *$ & 0 & 0 & $1 * * *$ & 0 & 0 & $1 * * *$ & 0 \\
\hline 8471 & 0 & $1^{*}$ & 0 & 0 & 0 & 1 & 0 & 0 & 1 & 0 & 0 & 1 \\
\hline 8483 & - & - & - & - & - & - & - & - & - & 0 & 0 & 1 \\
\hline 8501 & 0 & 0 & 1 & 0 & 0 & 1 & 0 & 0 & 1 & 0 & 0 & 1 \\
\hline 8504 & 0 & 0 & 1 & 0 & 0 & 1 & 0 & 0 & 1 & 0 & $1 * *$ & 0 \\
\hline 8517 & 0 & 0 & 1 & 0 & $1 * *$ & 0 & 0 & 0 & 1 & 0 & 0 & 1 \\
\hline 8802 & 0 & 0 & 1 & 0 & 0 & 1 & 0 & 0 & 1 & 0 & $1 * *$ & 0 \\
\hline Total & 3 & 9 & 29 & 3 & 8 & 35 & 8 & 8 & 28 & 8 & 12 & 27 \\
\hline
\end{tabular}

Nota: $\left({ }^{* *}\right),\left(^{* *}\right)$ e $\left(^{*}\right)$ denotam o nível de significância a 1, 5 e $10 \%$ respectivamente.

Fonte: Elaboração própria.

Constata-se que sempre quando há cointegração entre um determinado setor e mais de uma medida de volatilidade do câmbio os sinais dos coeficientes de longo prazo da volatilidade são semelhantes, independentemente de considerar medidas lineares ou não lineares. ${ }^{7}$ Isto é, para esses setores, independentemente da medida de volatilidade,

7 Isso não ocorreu apenas no setor hidróxidos (2825). 
observa-se um padrão da relação entre volatilidade do câmbio e variações no valor exportado, indicando evidências favoráveis à robustez dos modelos estimados.

Nota-se que os modelos com medidas não lineares produziram mais relações de cointegração com volatilidade significativa: 16 para o $\operatorname{TGARCH}(1,1)$ e 20 para o EGARCH(1,1), contra 12 do desvio padrão móvel 6 e 11 do $\operatorname{GARCH}(1,1)$. Parte desta superioridade deve-se aos modelos captarem mais relações de cointegração quando a volatilidade do câmbio tem um efeito positivo nas exportações: 8 para o $\operatorname{TGARCH}(1,1)$ e 8 para o $\operatorname{EGARCH}(1,1)$, ao passo que são 3 para o desvio padrão móvel 6 e 3 para o GARCH $(1,1)$.

Para investigar se existe algum padrão específico nos setores de exportação, utiliza-se a distinção por Classificação Uniforme para o Comércio Internacional (CUCI). A Tabela 3 apresenta os resultados dos coeficientes de longo prazo entre as volatilidades do câmbio e os setores classificados pela sua seção da CUCI. Nota-se similaridade nos resultados com as diferentes medidas de volatilidade do câmbio e, uma similaridade ainda maior entre as medidas lineares (Desvio padrão móvel 6 e GARCH $(1,1)$ ) e entre as medidas não lineares (TGARCH( $(1,1)$ e EGARCH $(1,1))$.

Tabela 3 - Resultados dos coeficientes das volatilidades

\begin{tabular}{|c|c|c|c|c|c|c|c|c|c|c|c|c|}
\hline \multirow[t]{2}{*}{ Seção } & \multicolumn{3}{|c|}{$\begin{array}{l}\text { Desvio Padrão } \\
\text { móvel } 6\end{array}$} & \multicolumn{3}{|c|}{$\operatorname{GARCH}(1,1)$} & \multicolumn{3}{|c|}{$\operatorname{TGARCH}(1,1)$} & \multicolumn{3}{|c|}{$\operatorname{EGARCH}(1,1)$} \\
\hline & $\mathrm{P}$ & $\mathrm{N}$ & NA & $\mathrm{P}$ & $\mathrm{N}$ & NA & $\mathrm{P}$ & $\mathrm{N}$ & NA & $\mathrm{P}$ & $\mathrm{N}$ & NA \\
\hline $\begin{array}{l}\text { 0. Produtos alimentícios e } \\
\text { animais vivos }\end{array}$ & 0 & 0 & 4 & 0 & 0 & 4 & 0 & 0 & 4 & 0 & 0 & 4 \\
\hline 1. Bebidas e tabaco & 0 & 1 & 4 & 0 & 1 & 4 & 0 & 1 & 4 & 0 & 0 & 5 \\
\hline $\begin{array}{l}\text { 2. Materiais em bruto, não } \\
\text { comestíveis, exceto } \\
\text { combustíveis }\end{array}$ & 1 & 3 & 10 & 0 & 2 & 12 & 2 & 0 & 12 & 2 & 1 & 11 \\
\hline $\begin{array}{l}\text { 3. Combustíveis minerais, } \\
\text { lubrificantes e materiais } \\
\text { relacionados }\end{array}$ & 1 & 1 & 4 & 1 & 1 & 4 & 1 & 1 & 4 & 1 & 1 & 4 \\
\hline $\begin{array}{l}\text { 4. Óleos animais e } \\
\text { vegetais, gorduras e ceras }\end{array}$ & 1 & 0 & 10 & 1 & 0 & 10 & 2 & 1 & 8 & 2 & 1 & 8 \\
\hline $\begin{array}{l}\text { 5. Produtos químicos e } \\
\text { relacionados da Nova } \\
\text { Política Econômica (NEP) }\end{array}$ & 0 & 0 & 1 & 0 & 0 & 1 & 1 & 0 & 0 & 1 & 0 & 0 \\
\hline $\begin{array}{l}\text { 6. Artigos manufaturados, } \\
\text { classificados } \\
\text { principalmente } \\
\text { pelo material }\end{array}$ & 0 & 1 & 5 & 0 & 0 & 6 & 0 & 1 & 5 & 0 & 2 & 4 \\
\hline $\begin{array}{l}\text { 7. Máquinas e } \\
\text { equipamentos } \\
\text { de transporte }\end{array}$ & 0 & 0 & 14 & 1 & 1 & 12 & 2 & 1 & 11 & 2 & 2 & 10 \\
\hline
\end{tabular}


Tabela 3 - Resultados dos coeficientes das volatilidades (CONTINUAÇÃO)

\begin{tabular}{|c|c|c|c|c|c|c|c|c|c|c|c|c|}
\hline \multirow[t]{2}{*}{ Seção } & \multicolumn{3}{|c|}{$\begin{array}{l}\text { Desvio Padrão } \\
\text { móvel } 6\end{array}$} & \multicolumn{3}{|c|}{$\operatorname{GARCH}(1,1)$} & \multicolumn{3}{|c|}{$\operatorname{TGARCH}(1,1)$} & \multicolumn{3}{|c|}{$\operatorname{EGARCH}(1,1)$} \\
\hline & $P$ & $\mathrm{~N}$ & NA & $\mathrm{P}$ & $\mathrm{N}$ & NA & $\mathrm{P}$ & $\mathrm{N}$ & NA & $\mathrm{P}$ & $\mathrm{N}$ & NA \\
\hline 8. Obras diversas & 0 & 3 & 22 & 0 & 3 & 22 & 0 & 3 & 22 & 0 & 5 & 20 \\
\hline $\begin{array}{l}\text { 9. Mercadorias e } \\
\text { transações não } \\
\text { especificadas, em outras } \\
\text { partes da CUCI }\end{array}$ & 0 & 0 & 4 & 0 & 0 & 4 & 0 & 0 & 4 & 0 & 0 & 4 \\
\hline Todos as seções & 3 & 9 & 78 & 3 & 8 & 79 & 8 & 8 & 74 & 8 & 12 & 70 \\
\hline
\end{tabular}

Nota: P - positivo, N - Negativo, NA - Não afetado.

Fonte: Elaboração própria.

Como apenas cerca de $16,5 \%$ dos setores respondem no longo prazo à volatilidade do câmbio (com um coeficiente positivo ou negativo), não se esperam grandes efeitos em qualquer setor específico. O resultado para os setores de produtos alimentícios e animais vivos é consoante, em certa medida, ao encontrado em Corrêa, Vasconcelos e Lima Junior (2018), para os quais não há a relação de longo prazo para produtos básicos exportados para os Estados Unidos.

A porcentagem de coeficientes que são positivos ou negativos dentro de um único setor deve ter um efeito ainda menor. Contudo, se um setor tiver um comportamento que aproxima dessas ações, pode-se utilizar isso como evidência de que ele se comporta de maneira única e potencialmente interessante.

A seção obras diversas apresentou uma parcela considerável dos setores com coeficientes negativos para todas as medidas de volatilidade, o que sugere um setor vulnerável à volatilidade do câmbio. Dois setores que tiveram parcela um pouco menor do que a do anterior, mas com sinal do efeito heterogêneo, foram: máquinas e equipamentos de transporte; e materiais em bruto, não comestíveis, exceto combustíveis. Já a seção de óleos animais e vegetais, gorduras e ceras tem parcela considerável de setores com coeficientes positivos, principalmente para medidas não lineares de volatilidade.

Ao analisar individualmente os setores, verifica-se que o sinal dos coeficientes de longo prazo da volatilidade do câmbio e suas características específicas são consoantes a teoria econômica. Segundo as hipóteses de Giovannini (1988), Klein (1990), Aghion et al. (2009), os setores em que a relação do longo prazo se apresenta negativamente estão relacionados a produtos com elevada dependência do capital externo, tais como veículos aéreos, veículos espaciais, máquinas para construção civil, ferro fundido bruto e hidrocarbonetos cíclicos. Desse modo, a incerteza do câmbio impossibilita a atração de capitais e restringe a dinâmica dos investimentos. Outra possibilidade são os setores com demanda inelástica, cuja neutralidade ao risco das empresas exportadoras levaria a cortes de preços em períodos de 
elevada volatilidade e, por consequência, queda do valor exportado. São eles: açúcares de cana ou de beterraba, sumo de frutas, gelatinas e seus derivados, couros e calçados.

Por fim, os setores econômicos positivamente afetados no longo prazo pela volatilidade do câmbio são produtos essencialmente pouco dependentes do capital externo, tais como: álcool etílico não desnaturado, óleos essenciais, pastas químicas de madeira. Aqueles com demanda altamente elástica também demonstram o mesmo comportamento frente à volatilidade do câmbio a longo prazo, como por exemplo: produtos semimanufaturados de ferro ou aço e pedras preciosas ou semipreciosas.

\section{CONSIDERAÇÕES FINAIS}

Desde o colapso do sistema de Bretton Woods, em 1971, a volatilidade da taxa de câmbio vem sendo uma das principais variáveis para determinar o comércio internacional. Embora se possa pensar que quanto mais volátil a taxa de câmbio menor os fluxos comerciais, alguns trabalhos empíricos recentes apresentaram evidências de que muitos produtos não são afetados pela incerteza cambial e que certos produtos respondem positivamente.

Deste modo, este artigo buscou identificar as relações de longo prazo entre a volatilidade da taxa de câmbio real (R \$ U \$) e as exportações brasileiras para os Estados Unidos. Para tanto, aplicou-se a metodologia de cointegração baseada no teste de fronteira de Pesaran em quatro medidas de volatilidade da taxa de câmbio com os cem principais setores de exportação a quatro dígitos da NCM entre Brasil e Estados Unidos no período de janeiro de 1999 a fevereiro de 2017.

Os resultados encontrados se mostraram robustos, uma vez que: i) 37 setores apresentaram relação de longo prazo com todas as medidas de volatilidade do câmbio; e ii) quando mais de uma medida de volatilidade foi significativa na relação cointegrante, sempre houve concordância com o sinal desta relação. Ressalta-se que aproximadamente $16,5 \%$ dos setores respondem no longo prazo à volatilidade do câmbio, sendo similar à porcentagem encontrada por Bahmani-Oskooee, Harvey e Hegerty (2013), de 23\%.

Adicionalmente, este trabalho contribui para a literatura referente ao tema na aplicação de quatro de medidas de volatilidade para a taxa de câmbio, sendo elas duas lineares e duas não lineares. A grande vantagem das duas últimas é que elas permitem que choques positivos e negativos possam afetar a medida de volatilidade com pesos diferentes. As medidas não lineares propostas indicaram que choques positivos no câmbio implicam em maior volatilidade para os períodos seguintes. Os resultados dos testes de Pesaran se mostraram similares para as diferentes medidas de volatilidade, contudo, notou-se que modelos considerando medidas não lineares de volatilidade 
cambial obtiveram mais relações de longo prazo positivas do que os modelos com medidas lineares. Uma possível justificativa é o fato de as depreciações cambiais terem papel mais relevante para determinar o grau de incerteza dos agentes em relação aos movimentos do câmbio, quando comparado às apreciações.

Para buscar padrões dos impactos da volatilidade cambial nos fluxos comerciais foram examinadas as exportações pela classificação da CUCI, sendo encontrados três padrões principais: i) a seção de obras diversas é a mais afetada negativamente, mostrando ser uma seção frágil a variações do câmbio, ii) as seções de máquinas e equipamentos de transporte e, materiais em bruto exceto combustíveis, são seções afetadas heterogeneamente pela volatilidade cambial, dependendo especificamente do setor, e iii) a seção de óleos animais e vegetais, gorduras e ceras é a seção mais afetada positivamente, ou seja, variações na taxa de câmbio aumentam o fluxo comercial dos produtos desta seção.

Analisando o sinal da relação de longo prazo entre a volatilidade da taxa de câmbio e os setores das exportações Brasil/EUA, os resultados corroboram os de BahmaniOskooee, Harvey e Hegerty (2013), sugerindo que as relações de longo prazo positivas entre o fluxo de comércio e a volatilidade cambial superam as negativas, indicando que este padrão pode ser característico das relações comerciais entre Brasil/EUA. Os setores negativamente relacionados com a volatilidade da taxa de câmbio são produtos com elevada dependência do capital externo ou com característica de demanda inelástica. Enquanto os positivamente relacionados são produtos com pouca dependência do capital externo ou com demanda altamente elástica.

\section{REFERÊNCIAS}

AFTAB, M.; SYED, K. B. S.; KATPER, N. A. Exchange-rate volatility and Malaysian-Thai bilateral industry trade flows. Journal of Economic Studies, v. 44, n. 1, p. 99-114, 2017. DOI: 10.1108/ JES-05-2015-0091.

AGHION, P; BACCHETTA, P; RANCIÈRE, R; ROGOFF, K. Exchange rate volatility and productivity growth: The role of financial development. Journal of Monetary Economics, v. 56, n. 4, p. 494-513, 2009.

AGUIRRE, A.; FERREIRA, A.; NOTINI, H. The impact of exchange rate volatility on Brazilian manufactured exports. Económica, v. 53, n. 1-2, p. 3-19, 2007.

ARIZE, A. C.; OSANG, T.; SLOTTJE, D. J. Exchange-rate volatility in Latin America and its impact on foreign trade. International Review of Economics \& Finance, v. 17, n. 1, p. 33-44, 2008.

ASSEERY, A.; PEEL, D. A. The effects of exchange rate volatility on exports: Some new estimates. Economics Letters, v. 37, p. 173-177, 1991.

BAHMANI-OSKOOEE, M.; HEGERTY, S. W. Exchange rate volatility and trade flows: A review article. Journal of Economic Studies, v. 34, n. 3, p. 211-255, 2007. 
BAHMANI-OSKOOEE, M.; HARVEY, H.; HEGERTY, S. W. The effects of exchange-rate volatility on commodity trade between the U.S. and Brazil. The North American Journal of Economics and Finance, v. 25, p. 70-93, 2013.

BAILEY, M. J.; TAVLAS, G. S.; ULAN, M. The impact of exchange-rate volatility on export growth: Some theoretical considerations and empirical results. Journal of Policy Modeling, v. 9, n. 1, p. 225-243, 1987.

BANERJEE, A.; DOLADO, J. J.; MESTRE, R. Error-correction mechanism tests for cointegration in a single-equation framework. Journal of Time Series Analysis, v. 19, n. 3, p. 267-283, 1998.

BITTENCOURT, G. M.; CAMPOS, A. C. Efeitos da instabilidade da taxa de câmbio no comércio setorial entre Brasil e seus principais parceiros comerciais. Economia Aplicada, v. 18, n. 4, p. 657-678, 2014.

BITTENCOURT, M. V. L.; LARSON, D. W.; THOMPSON, S. R. Impactos da volatilidade da taxa de câmbio no comércio setorial do mercosul. Estudos Econômicos, v. 37, n. 4, p. 791-816, out./dez. 2007.

BOARD OF GOVERNORS OF THE FEDERAL RESERVE SYSTEM. Industrial Production: Total Index (INDPRO). St Louis: Federal Reserve Bank of St. Louis, 2017. Acesso em Maio de 2017.

BOLLERSLEV, T.; CHOU, R. Y.; KRONER, K. F. ARCH modeling in finance: A review of the theory and empirical evidence. Journal of Econometrics, v. 52, n. 1-2, p. 5-59, 1992.

BRASIL. Ministério do Desenvolvimento, Indústria e Comércio Exterior. Secretaria de Comércio Exterior. Sistema de Análise das Informações de Comércio Exterior via Internet: AliceWeb/ Comexstat. Volume de exportações do Brasil para os EUA. MDIC, 2017. Acesso em: mai. 2017.

BRODSKY, D. A. Fixed versus flexible exchange rates and the measurement of exchange rate instability. Journal of International Economics, v. 16, v. 3-4, p. 295-306, 1984.

BROOKS, C. Introductory econometrics for finance. Cambridge, UK: Cambridge University Press, 2008.

BUENO, R. L. S. Econometria de séries temporais. São Paulo: Cengage Learning, 2011.

COES, D. V. The crawling peg and exchange rate uncertainty. In: WILLIAMSON, J. (Ed.) Exchange rate rules: The theory, performance, and prospect of the crawling peg. New York, NY: St Martins Press, 1981, p. 113-139.

CORRÊA K. D.; VASCONCELOS, C. R. F.; LIMA JÚNIOR, L. A. Volatilidade da taxa de câmbio real efetiva e exportações brasileiras. Análise Econômica, v. 36, n. 70, p. 83-119, jun. 2018.

CÔTÉ, A. Exchange rate volatility and trade. Working Paper, n. 94-95, 1994.

DE GRAUWE, P. Exchange rate variability and the slowdown in growth of international trade. IMF Staff Papers, v. 35, n. 1. p. 63-84.

DE VITA, G.; ABBOTT, A. The impact of exchange rate volatility on UK exports to EU countries. Scottish Journal of Political Economy, v. 51, n. 1, p. 62-81, 2004.

ENGLE, R. F. Autoregessive conditional heteroscedasticity with estimates of the variance of United Kingdom inflation. Econometrica, v. 50, n. 4, p. 987-1008, jul. 1982.

ENGLE, R. F.; GRANGER, C. W. J. Co-integration and error correction: Representation, estimation, and testing. Econometrica, v. 55, n. 2, p. 251-276, mar. 1987. 
ESQUIVEL, G.; LARRAÍN, F. B. The impact of G-3 exchange rate volatility on developing countries. UNCTAD G-24 Discussion Paper Series, n. 16, jan. 2002.

GIOVANNINI, A. Exchange rates and traded goods prices. Journal of international Economics, v. 24 , p. $45-68,1988$.

GOLDSTEIN, M.; KHAN, M. S. Income and price effects in foreign trade. In: JONES, R. W.; KENEN, P. B. (Eds.). Handbook of International Economics. Amsterdam: North-Holland, 1985, p. 1041-1105.

GONZAGA, G. M.; TERRA, M. C. T. Equilibrium real exchange rate, volatility, and stabilization. Journal of Development Economics, v. 54, n. 1, p. 77-100, 1997.

GRIEBELER, M. Models for forecasting exchange rate volatility: A comparison between developed and emerging countries. Economics Bulletin, v. 34, n. 3, p. 1618-1630, 2014.

GRIER, K. B.; SMALLWOOD, A. D. Uncertainty and export performance: Evidence from 18 countries. Journal of Money, Credit and Banking, v. 39, n. 4, p. 965-979, 2007.

HÉRICOURT, J.; PONCET, S. Exchange rate volatility, financial constraints, and trade: Empirical evidence from Chinese firms. The World Bank Economic Review, v. 29, n. 3, p. 550-578, 2013.

HOOPER, P.; KOHLHAGEN, S. W. The effects of exchange rate uncertainty on the prices and volume of international trade. Journal of International Economics, v. 8, n. 4, p. 483-511, 1978.

IPEA - INSTITUTO DE PESQUISA ECONÔMICA APLICADA. Taxa de câmbio real bilateral. Dados macroeconômicos e regionais.

JOHANSEN, S. Statistical analysis of cointegration vectors. Journal of Economic Dynamic and Control, v. 12, n. 2-3, p. 231-254, 1988.

JOHANSEN, S. Estimation and hypothesis testing of cointegration vectors in Gaussian vector autoregressive models. Econometrica, v. 59, n. 6, p. 1551-1580, nov. 1991.

KAFLE, K. R. Exchange rate volatility and bilateral agricultural trade flows: The case of the Unite States and OECD countries. 2011. Thesis (Master of Science) - The Department of Agricultural Economics and Agribusiness, Lousiana State University, Baton Rouge, 2011.

KENEN, P. B.; RODRIK, D. Measuring and analyzing the effects of short-term volatility in real exchange rates. The Review of Economics and Statistics, v. 68, n. 2, p. 311-315, 1986.

KIM, C. B. Does exchange rate volatility affect Korea's seaborne import volume? The Asian Journal of Shipping and Logistics, v. 33, n. 1, p. 43-50, 2017. Doi: 10.1016/j.ajsl.2017.03.006.

KLEIN, M. W. Sectoral effects of exchange rate volatility on united state exports. Journal of International Money and Finance, v. 9, n. 3, p. 299-308, 1990.

MCKENZIE, M. D. The impact of exchange rate volatility on international trade flows. Journal of Economic Surveys, v. 13, n. 1, p. 71-104, 1999.

MEDHORA, R. The effect of exchange rate variability on trade: The case of the West African monetary union's imports. World Development, v. 18, n. 2, p. 313-324, 1990.

PESARAN, M. H.; SHIN, Y.; SMITH, R. J. Bounds testing approaches to the analysis of level relationships. Journal of Applied Econometrics, v. 16, n. 3, p. 289-326, 2001. 
SHAIKH, S, A; HONGBING, O. Exchange rate volatility and trade flows: Evidence from China, Pakistan and India. International Journal of Economics and Finance, v. 7, n. 11, p. 121-217, 2015.

SILVA, F. A.; FREITAS, C. O.; MATTOS, L. B. Volatilidade da taxa de câmbio e seus efeitos sobre o fluxo de comércio dos países da América do Sul. Revista de Economia Contemporânea, v. 20, n, 2, p. 229-249, mai./ago. 2016.

VERHEYEN, F. Bilateral exports from euro zone countries to the US-Does exchange rate variability play a role? International Review of Economics \& Finance, v. 24, p. 97-108, 2012. 


\section{APÊNDICE}

Tabela I - Testes de cointegração

\begin{tabular}{|c|c|c|c|c|c|c|c|c|c|c|c|c|}
\hline \multirow[b]{2}{*}{ Setor } & \multicolumn{3}{|c|}{ Desvio padrão móvel 6} & \multicolumn{3}{|c|}{$\operatorname{GARCH}(1,1)$} & \multicolumn{3}{|c|}{ TGARCH $(1,1)$} & \multicolumn{3}{|c|}{ EGARCH $(1,1)$} \\
\hline & $F$ test & $\begin{array}{c}\text { ECM } \\
\text { t-1 }\end{array}$ & $\begin{array}{c}\text { Coint } \\
?\end{array}$ & $F$ test & $\begin{array}{c}\text { ECM } \\
t-1\end{array}$ & $\begin{array}{c}\text { Coint } \\
?\end{array}$ & $\mathrm{~F}$ test & $\begin{array}{c}\text { ECM } \\
\text { t-1 }\end{array}$ & $\begin{array}{c}\text { Coint } \\
?\end{array}$ & F test & $\begin{array}{c}\text { ECM } \\
t-1\end{array}$ & $\begin{array}{c}\text { Coint } \\
?\end{array}$ \\
\hline 409 & 2,90 & $-3,04$ & $\mathrm{~N}$ & 2,69 & $-2,90$ & $\mathrm{~N}$ & 2,68 & $-2,88$ & $\mathrm{~N}$ & 2,68 & $-2,90$ & $\mathrm{~N}$ \\
\hline 801 & 2,63 & $-1,70$ & $\mathrm{~N}$ & 1,97 & $-1,60$ & $\mathrm{~N}$ & 1,90 & $-1,40$ & $\mathrm{~N}$ & 1,81 & $-1,40$ & $\mathrm{~N}$ \\
\hline 901 & 6,86 & $-5,15$ & $\mathrm{~S}$ & 8,15 & $-5,70$ & $\mathrm{~S}$ & 7,73 & $-5,28$ & S & 6,23 & $-4,61$ & $\mathrm{~S}$ \\
\hline 904 & 14,30 & $-7,50$ & $\mathrm{~S}$ & 13,95 & $-7,41$ & $\mathrm{~S}$ & 14,15 & $-7,43$ & $\mathrm{~S}$ & 15,23 & $-7,71$ & $\mathrm{~S}$ \\
\hline 1005 & 4,95 & $-3,85$ & $\mathrm{~S}$ & 5,45 & $-3,95$ & S & 4,90 & $-3,80$ & $\mathrm{~N}$ & 4,95 & $-3,81$ & $\mathrm{~N}$ \\
\hline 1602 & 4,04 & $-4,14$ & $\mathrm{~S}$ & 4,11 & $-4,14$ & $\mathrm{~S}$ & 4,14 & $-4,18$ & $\mathrm{~S}$ & 4,10 & $-4,17$ & $\mathrm{~S}$ \\
\hline 1701 & 43,11 & $-11,32$ & $\mathrm{~S}$ & 60,20 & $-13,32$ & $\mathrm{~S}$ & 31,47 & $-9,70$ & $\mathrm{~S}$ & 35,64 & $-10,29$ & $\mathrm{~S}$ \\
\hline 1704 & 2,95 & $-2,64$ & $\mathrm{~N}$ & 1,48 & $-2,16$ & $\mathrm{~N}$ & 1,60 & $-2,15$ & $\mathrm{~N}$ & 1,75 & $-2,19$ & $\mathrm{~N}$ \\
\hline 1804 & 3,31 & $-3,50$ & $\mathrm{~N}$ & 4,11 & $-3,96$ & $\mathrm{~S}$ & 2,10 & $-2,67$ & $\mathrm{~N}$ & 2,14 & $-2,55$ & $\mathrm{~N}$ \\
\hline 2009 & 12,63 & $-6,97$ & S & 20,20 & $-8,94$ & $\mathrm{~S}$ & 14,54 & $-7,26$ & $\mathrm{~S}$ & 14,60 & $-7,37$ & $\mathrm{~S}$ \\
\hline 2101 & 7,00 & $-5,17$ & $\mathrm{~S}$ & 7,57 & $-5,30$ & $\mathrm{~S}$ & 7,65 & $-4,95$ & $\mathrm{~S}$ & 7,70 & $-4,90$ & $\mathrm{~S}$ \\
\hline 2207 & 12,58 & $-7,05$ & S & 12,65 & $-7,11$ & S & 13,08 & $-7,16$ & S & 12,78 & $-7,19$ & S \\
\hline 2401 & 34,91 & $-11,77$ & $\mathrm{~S}$ & 35,07 & $-12,41$ & $\mathrm{~S}$ & 35,19 & $-12,41$ & $\mathrm{~S}$ & 34,41 & $-11,72$ & $\mathrm{~S}$ \\
\hline 2507 & 1,93 & $-1,08$ & $\mathrm{~N}$ & 1,76 & $-1,67$ & $\mathrm{~N}$ & 2,13 & $-1,65$ & $\mathrm{~N}$ & 2,18 & $-1,74$ & $\mathrm{~N}$ \\
\hline 2606 & 1,73 & $-1,70$ & $\mathrm{~N}$ & 1,40 & $-1,78$ & $\mathrm{~N}$ & 1,39 & $-1,55$ & $\mathrm{~N}$ & 1,39 & $-1,48$ & $\mathrm{~N}$ \\
\hline 2709 & 3,31 & $-3,22$ & $\mathrm{~N}$ & 8,28 & $-5,84$ & $\mathrm{~S}$ & 7,91 & $-5,68$ & $\mathrm{~S}$ & 7,15 & $-5,41$ & $\mathrm{~S}$ \\
\hline 2710 & 13,05 & $-7,26$ & $\mathrm{~S}$ & 13,37 & $-7,43$ & $\mathrm{~S}$ & 11,84 & $-6,88$ & $\mathrm{~S}$ & 11,28 & $-6,72$ & $\mathrm{~S}$ \\
\hline 2804 & 8,21 & $-5,42$ & $\mathrm{~S}$ & 5,40 & $-4,73$ & $\mathrm{~S}$ & 5,43 & $-4,68$ & $\mathrm{~S}$ & 5,68 & $-4,41$ & $\mathrm{~S}$ \\
\hline 2818 & 3,32 & $-1,80$ & $\mathrm{~N}$ & 3,19 & $-1,80$ & $\mathrm{~N}$ & 3,15 & $-1,75$ & $\mathrm{~N}$ & 3,08 & $-1,75$ & $\mathrm{~N}$ \\
\hline 2825 & 3,72 & $-3,48$ & $\mathrm{~N}$ & 6,53 & $-4,25$ & $\mathrm{~S}$ & 5,76 & $-4,19$ & S & 5,01 & $-3,31$ & S \\
\hline 2901 & 2,27 & $-2,37$ & $\mathrm{~N}$ & 3,02 & $-2,63$ & $\mathrm{~N}$ & 2,79 & $-2,37$ & $\mathrm{~N}$ & 2,67 & $-2,63$ & $\mathrm{~N}$ \\
\hline 2902 & 7,70 & $-5,49$ & $\mathrm{~S}$ & 3,38 & $-3,52$ & $\mathrm{~N}$ & 8,42 & $-4,14$ & $\mathrm{~S}$ & 4,13 & $-3,82$ & $\mathrm{~N}$ \\
\hline 2922 & 12,25 & $-6,95$ & $\mathrm{~S}$ & 7,62 & $-4,60$ & $\mathrm{~S}$ & 6,79 & $-4,75$ & $\mathrm{~S}$ & 6,47 & $-4,67$ & $\mathrm{~S}$ \\
\hline 409 & 2,90 & $-3,04$ & $\mathrm{~N}$ & 2,69 & $-2,90$ & $\mathrm{~N}$ & 2,68 & $-2,88$ & $\mathrm{~N}$ & 2,37 & $-2,21$ & $\mathrm{~N}$ \\
\hline 801 & 2,63 & $-1,70$ & $\mathrm{~N}$ & 1,97 & $-1,60$ & $\mathrm{~N}$ & 1,90 & $-1,40$ & $\mathrm{~N}$ & 19,23 & $-7,47$ & $\mathrm{~S}$ \\
\hline 901 & 6,86 & $-5,15$ & $\mathrm{~S}$ & 8,15 & $-5,70$ & $\mathrm{~S}$ & 7,73 & $-5,28$ & $\mathrm{~S}$ & 13,93 & $-7,50$ & S \\
\hline 904 & 14,30 & $-7,50$ & $\mathrm{~S}$ & 13,95 & $-7,41$ & $\mathrm{~S}$ & 14,15 & $-7,43$ & $\mathrm{~S}$ & 29,15 & $-11,07$ & $\mathrm{~S}$ \\
\hline 1005 & 4,95 & $-3,85$ & $\mathrm{~S}$ & 5,45 & $-3,95$ & $\mathrm{~S}$ & 4,90 & $-3,80$ & $\mathrm{~N}$ & 2,30 & $-2,85$ & $\mathrm{~N}$ \\
\hline 1602 & 4,04 & $-4,14$ & $\mathrm{~S}$ & 4,11 & $-4,14$ & $\mathrm{~S}$ & 4,14 & $-4,18$ & $\mathrm{~S}$ & 2,27 & $-0,12$ & $\mathrm{~N}$ \\
\hline 1701 & 43,11 & $-11,32$ & $\mathrm{~S}$ & 60,20 & $-13,32$ & $\mathrm{~S}$ & 31,47 & $-9,70$ & $\mathrm{~S}$ & 5,43 & $-5,00$ & $\mathrm{~S}$ \\
\hline 1704 & 2,95 & $-2,64$ & $\mathrm{~N}$ & 1,48 & $-2,16$ & $\mathrm{~N}$ & 1,60 & $-2,15$ & $\mathrm{~N}$ & 1,60 & $-2,53$ & $\mathrm{~N}$ \\
\hline 1804 & 3,31 & $-3,50$ & $\mathrm{~N}$ & 4,11 & $-3,96$ & $\mathrm{~S}$ & 2,10 & $-2,67$ & $\mathrm{~N}$ & 2,14 & $-2,55$ & $\mathrm{~N}$ \\
\hline 2009 & 12,63 & $-6,97$ & $\mathrm{~S}$ & 20,20 & $-8,94$ & $\mathrm{~S}$ & 14,54 & $-7,26$ & $\mathrm{~S}$ & 14,60 & $-7,37$ & $\mathrm{~S}$ \\
\hline 2101 & 7,00 & $-5,17$ & $\mathrm{~S}$ & 7,57 & $-5,30$ & $\mathrm{~S}$ & 7,65 & $-4,95$ & $\mathrm{~S}$ & 7,70 & $-4,90$ & $\mathrm{~S}$ \\
\hline
\end{tabular}

(continua) 
TABELA I (CONTINUAÇÃO)

\begin{tabular}{|c|c|c|c|c|c|c|c|c|c|c|c|c|}
\hline \multirow[b]{2}{*}{ Setor } & \multicolumn{3}{|c|}{ Desvio padrão móvel 6} & \multicolumn{3}{|c|}{$\operatorname{GARCH}(1,1)$} & \multicolumn{3}{|c|}{ TGARCH $(1,1)$} & \multicolumn{3}{|c|}{ EGARCH $(1,1)$} \\
\hline & F test & $\begin{array}{c}\text { ECM } \\
\text { t-1 }\end{array}$ & $\begin{array}{c}\text { Coint } \\
?\end{array}$ & F test & $\begin{array}{c}\text { ECM } \\
\text { t-1 }\end{array}$ & $\begin{array}{c}\text { Coint } \\
?\end{array}$ & F test & $\begin{array}{c}\text { ECM } \\
\text { t-1 }\end{array}$ & $\begin{array}{c}\text { Coint } \\
?\end{array}$ & F test & $\begin{array}{c}\text { ECM } \\
\text { t-1 }\end{array}$ & $\begin{array}{c}\text { Coint } \\
?\end{array}$ \\
\hline 2207 & 12,58 & $-7,05$ & $\mathrm{~S}$ & 12,65 & $-7,11$ & $\mathrm{~S}$ & 13,08 & $-7,16$ & $\mathrm{~S}$ & 12,78 & $-7,19$ & S \\
\hline 2401 & 34,91 & $-11,77$ & $\mathrm{~S}$ & 35,07 & $-12,41$ & $\mathrm{~S}$ & 35,19 & $-12,41$ & $\mathrm{~S}$ & 34,41 & $-11,72$ & $\mathrm{~S}$ \\
\hline 2507 & 1,93 & $-1,08$ & $\mathrm{~N}$ & 1,76 & $-1,67$ & $\mathrm{~N}$ & 2,13 & $-1,65$ & $\mathrm{~N}$ & 2,18 & $-1,74$ & $\mathrm{~N}$ \\
\hline 2606 & 1,73 & $-1,70$ & $\mathrm{~N}$ & 1,40 & $-1,78$ & $\mathrm{~N}$ & 1,39 & $-1,55$ & $\mathrm{~N}$ & 1,39 & $-1,48$ & $\mathrm{~N}$ \\
\hline 2709 & 3,31 & $-3,22$ & $\mathrm{~N}$ & 8,28 & $-5,84$ & $\mathrm{~S}$ & 7,91 & $-5,68$ & $\mathrm{~S}$ & 7,15 & $-5,41$ & $\mathrm{~S}$ \\
\hline 2710 & 13,05 & $-7,26$ & $\mathrm{~S}$ & 13,37 & $-7,43$ & $\mathrm{~S}$ & 11,84 & $-6,88$ & $\mathrm{~S}$ & 11,28 & $-6,72$ & $\mathrm{~S}$ \\
\hline 2804 & 8,21 & $-5,42$ & $\mathrm{~S}$ & 5,40 & $-4,73$ & $\mathrm{~S}$ & 5,43 & $-4,68$ & $\mathrm{~S}$ & 5,68 & $-4,41$ & $\mathrm{~S}$ \\
\hline 2818 & 3,32 & $-1,80$ & $\mathrm{~N}$ & 3,19 & $-1,80$ & $\mathrm{~N}$ & 3,15 & $-1,75$ & $\mathrm{~N}$ & 3,08 & $-1,75$ & $\mathrm{~N}$ \\
\hline 2825 & 3,72 & $-3,48$ & $\mathrm{~N}$ & 6,53 & $-4,25$ & $\mathrm{~S}$ & 5,76 & $-4,19$ & $S$ & 5,01 & $-3,31$ & $\mathrm{~S}$ \\
\hline 2901 & 2,27 & $-2,37$ & $\mathrm{~N}$ & 3,02 & $-2,63$ & $\mathrm{~N}$ & 2,79 & $-2,37$ & $\mathrm{~N}$ & 2,67 & $-2,63$ & $\mathrm{~N}$ \\
\hline 2902 & 7,70 & $-5,49$ & $\mathrm{~S}$ & 3,38 & $-3,52$ & $\mathrm{~N}$ & 8,42 & $-4,14$ & S & 4,13 & $-3,82$ & $\mathrm{~N}$ \\
\hline 2922 & 12,25 & $-6,95$ & $\mathrm{~S}$ & 7,62 & $-4,60$ & $\mathrm{~S}$ & 6,79 & $-4,75$ & $\mathrm{~S}$ & 6,47 & $-4,67$ & $\mathrm{~S}$ \\
\hline 3004 & 5,67 & $-1,35$ & $\mathrm{~N}$ & 6,54 & $-1,60$ & $\mathrm{~S}$ & 2,29 & $-2,19$ & $\mathrm{~N}$ & 2,37 & $-2,21$ & $\mathrm{~N}$ \\
\hline 3005 & 7,25 & $-4,80$ & $\mathrm{~S}$ & 19,30 & $-7,37$ & $\mathrm{~S}$ & 20,42 & $-7,65$ & $\mathrm{~S}$ & 19,23 & $-7,47$ & $\mathrm{~S}$ \\
\hline 3301 & 6,59 & $-4,49$ & S & 28,11 & $-10,47$ & $\mathrm{~S}$ & 15,46 & $-7,70$ & $\mathrm{~S}$ & 13,93 & $-7,50$ & $\mathrm{~S}$ \\
\hline 3503 & 26,68 & $-10,43$ & $\mathrm{~S}$ & 30,53 & $-10,98$ & $\mathrm{~S}$ & 28,85 & $-10,67$ & $\mathrm{~S}$ & 29,15 & $-11,07$ & $\mathrm{~S}$ \\
\hline 3901 & 2,05 & $-2,50$ & $\mathrm{~N}$ & 1,95 & $-2,60$ & $\mathrm{~N}$ & 2,30 & $-2,94$ & $\mathrm{~N}$ & 2,30 & $-2,85$ & $\mathrm{~N}$ \\
\hline 3907 & 0,80 & $-0,36$ & $\mathrm{~N}$ & 1,07 & $-0,31$ & $\mathrm{~N}$ & 2,30 & $-0,24$ & $\mathrm{~N}$ & 2,27 & $-0,12$ & $\mathrm{~N}$ \\
\hline 4002 & 6,01 & $-4,76$ & $\mathrm{~S}$ & 5,45 & $-4,70$ & $\mathrm{~S}$ & 5,42 & $-5,01$ & $\mathrm{~S}$ & 5,43 & $-5,00$ & $\mathrm{~S}$ \\
\hline 4011 & 1,50 & $-2,50$ & $\mathrm{~N}$ & 1,56 & $-2,54$ & $\mathrm{~N}$ & 1,56 & $-2,57$ & $\mathrm{~N}$ & 1,60 & $-2,53$ & $\mathrm{~N}$ \\
\hline 4107 & 7,61 & $-4,33$ & $\mathrm{~S}$ & 8,90 & $-4,94$ & $\mathrm{~S}$ & 9,73 & $-5,08$ & $\mathrm{~S}$ & 9,43 & $-5,05$ & $\mathrm{~S}$ \\
\hline 4205 & 1,00 & $-1,00$ & $\mathrm{~N}$ & 1,00 & $-1,01$ & $\mathrm{~N}$ & 1,00 & $-1,05$ & $\mathrm{~N}$ & 1,05 & $-1,10$ & $\mathrm{~N}$ \\
\hline 4407 & 4,11 & $-3,81$ & $\mathrm{~N}$ & 3,50 & $-3,51$ & $\mathrm{~N}$ & 3,43 & $-3,40$ & $\mathrm{~N}$ & 3,64 & $-3,44$ & $\mathrm{~N}$ \\
\hline 4409 & 1,79 & 0,49 & $\mathrm{~N}$ & 1,92 & 0,67 & $\mathrm{~N}$ & 1,96 & 0,64 & $\mathrm{~N}$ & 1,90 & 0,63 & $\mathrm{~N}$ \\
\hline 4411 & 2,66 & $-3,15$ & $\mathrm{~N}$ & 2,94 & $-3,08$ & $\mathrm{~N}$ & 3,30 & $-3,31$ & $\mathrm{~N}$ & 3,71 & $-3,56$ & $\mathrm{~N}$ \\
\hline 4412 & 4,33 & $-3,68$ & $\mathrm{~N}$ & 3,74 & $-3,43$ & $\mathrm{~N}$ & 3,70 & $-3,49$ & $\mathrm{~N}$ & 4,40 & $-3,90$ & $\mathrm{~S}$ \\
\hline 4418 & 1,37 & $-1,88$ & $\mathrm{~N}$ & 1,80 & $-1,88$ & $\mathrm{~N}$ & 1,92 & $-1,90$ & $\mathrm{~N}$ & 2,00 & $-1,80$ & $\mathrm{~N}$ \\
\hline 4703 & 7,48 & $-5,51$ & $\mathrm{~S}$ & 11,38 & $-6,54$ & $\mathrm{~S}$ & 9,18 & $-5,76$ & $S$ & 16,38 & $-8,02$ & $\mathrm{~S}$ \\
\hline 4802 & 4,42 & $-4,03$ & $\mathrm{~S}$ & 4,23 & $-3,95$ & $\mathrm{~S}$ & 4,20 & $-3,98$ & $\mathrm{~S}$ & 4,18 & $-4,04$ & $\mathrm{~S}$ \\
\hline 5607 & 30,16 & $-11,06$ & $\mathrm{~S}$ & 57,21 & $-15,01$ & $\mathrm{~S}$ & 57,95 & $-15,23$ & $\mathrm{~S}$ & 58,35 & $-15,31$ & S \\
\hline 6302 & 2,67 & $-1,55$ & $\mathrm{~N}$ & 2,32 & $-1,47$ & $\mathrm{~N}$ & 2,55 & $-1,60$ & $\mathrm{~N}$ & 3,30 & $-2,01$ & $\mathrm{~N}$ \\
\hline 6402 & 4,90 & $-4,25$ & $\mathrm{~S}$ & 4,40 & $-4,13$ & $\mathrm{~S}$ & 4,57 & $-4,13$ & $\mathrm{~S}$ & 4,70 & $-4,13$ & $\mathrm{~S}$ \\
\hline 6403 & 9,62 & $-3,48$ & $\mathrm{~S}$ & 7,88 & $-3,14$ & $\mathrm{~S}$ & 9,11 & $-2,96$ & $\mathrm{~S}$ & 9,80 & $-3,60$ & $\mathrm{~S}$ \\
\hline 6802 & 3,49 & $-3,62$ & $\mathrm{~N}$ & 3,06 & $-3,57$ & $\mathrm{~N}$ & 4,93 & $-3,58$ & $\mathrm{~S}$ & 5,23 & $-3,72$ & $\mathrm{~S}$ \\
\hline 6813 & 3,34 & $-1,74$ & $\mathrm{~N}$ & 4,01 & $-1,84$ & $\mathrm{~N}$ & 3,54 & $-1,94$ & $\mathrm{~N}$ & 3,26 & $-1,82$ & $\mathrm{~N}$ \\
\hline 6908 & 2,44 & $-1,81$ & $\mathrm{~N}$ & 3,05 & $-1,91$ & $\mathrm{~N}$ & 3,20 & $-1,82$ & $\mathrm{~N}$ & 3,08 & $-1,75$ & $\mathrm{~N}$ \\
\hline
\end{tabular}

(continua) 
TABELA I (CONTINUAÇÃO)

\begin{tabular}{|c|c|c|c|c|c|c|c|c|c|c|c|c|}
\hline \multirow[b]{2}{*}{ Setor } & \multicolumn{3}{|c|}{ Desvio padrão móvel 6} & \multicolumn{3}{|c|}{$\operatorname{GARCH}(1,1)$} & \multicolumn{3}{|c|}{ TGARCH $(1,1)$} & \multicolumn{3}{|c|}{$\operatorname{EGARCH}(1,1)$} \\
\hline & F test & $\begin{array}{c}\text { ECM } \\
\text { t-1 }\end{array}$ & $\begin{array}{c}\text { Coint } \\
?\end{array}$ & F test & $\begin{array}{c}\text { ECM } \\
\text { t-1 }\end{array}$ & $\begin{array}{c}\text { Coint } \\
?\end{array}$ & F test & $\begin{array}{c}\text { ECM } \\
t-1\end{array}$ & $\begin{array}{c}\text { Coint } \\
?\end{array}$ & F test & $\begin{array}{c}\text { ECM } \\
t-1\end{array}$ & $\begin{array}{c}\text { Coint } \\
?\end{array}$ \\
\hline 7103 & 52,42 & $-11,79$ & $\mathrm{~S}$ & 50,14 & $-14,23$ & $\mathrm{~S}$ & 50,81 & $-14,29$ & S & 50,99 & $-14,28$ & S \\
\hline 7108 & 1,79 & $-2,15$ & $\mathrm{~N}$ & 1,59 & $-2,00$ & $\mathrm{~N}$ & 3,67 & $-2,64$ & $\mathrm{~N}$ & 8,96 & $-4,11$ & $\mathrm{~S}$ \\
\hline 7201 & 0,66 & $-1,50$ & $\mathrm{~N}$ & 5,04 & $-4,10$ & $\mathrm{~S}$ & 5,05 & $-4,07$ & $\mathrm{~S}$ & 7,42 & $-4,29$ & $\mathrm{~S}$ \\
\hline 7202 & 17,64 & $-8,38$ & S & 29,48 & $-10,85$ & $\mathrm{~S}$ & 17,22 & $-8,27$ & $\mathrm{~S}$ & 17,28 & $-8,22$ & $\mathrm{~S}$ \\
\hline 7207 & 5,50 & $-4,60$ & $\mathrm{~S}$ & 5,41 & $-4,57$ & $\mathrm{~S}$ & 5,88 & $-4,72$ & $\mathrm{~S}$ & 6,21 & $-4,95$ & $\mathrm{~S}$ \\
\hline 7208 & 3,29 & $-3,35$ & $\mathrm{~N}$ & 3,37 & $-3,44$ & $\mathrm{~N}$ & 3,10 & $-3,20$ & $\mathrm{~N}$ & 3,48 & $-3,41$ & $\mathrm{~N}$ \\
\hline 7209 & 2,44 & $-3,11$ & $\mathrm{~N}$ & 2,40 & $-3,06$ & $\mathrm{~N}$ & 2,35 & $-3,02$ & $\mathrm{~N}$ & 2,35 & $-2,96$ & $\mathrm{~N}$ \\
\hline 7213 & 48,95 & $-14,01$ & $\mathrm{~S}$ & 49,94 & $-14,23$ & $\mathrm{~S}$ & 49,97 & $-14,12$ & $\mathrm{~S}$ & 49,97 & $-14,12$ & $\mathrm{~S}$ \\
\hline 7219 & 4,23 & $-3,69$ & $\mathrm{~N}$ & 4,02 & $-3,85$ & $\mathrm{~S}$ & 3,98 & $-3,83$ & $\mathrm{~S}$ & 6,80 & $-5,37$ & $\mathrm{~S}$ \\
\hline 7224 & 11,03 & $-6,51$ & $\mathrm{~S}$ & 11,01 & $-6,52$ & $\mathrm{~S}$ & 11,01 & $-6,52$ & $\mathrm{~S}$ & 11,22 & $-6,50$ & $\mathrm{~S}$ \\
\hline 7228 & 5,73 & $-4,58$ & $\mathrm{~S}$ & 6,05 & $-4,81$ & $\mathrm{~S}$ & 6,02 & $-4,84$ & $\mathrm{~S}$ & 5,72 & $-4,72$ & $\mathrm{~S}$ \\
\hline 7304 & 44,28 & $-13,41$ & S & 46,56 & $-13,65$ & $\mathrm{~S}$ & 47,30 & $-13,41$ & $\mathrm{~S}$ & 47,67 & $-13,56$ & $\mathrm{~S}$ \\
\hline 7326 & 2,11 & $-1,80$ & $\mathrm{~N}$ & 1,62 & $-1,92$ & $\mathrm{~N}$ & 2,01 & $-1,52$ & $\mathrm{~N}$ & 2,78 & $-2,08$ & $\mathrm{~N}$ \\
\hline 7606 & 2,39 & $-3,32$ & $\mathrm{~N}$ & 2,45 & $-2,65$ & $\mathrm{~N}$ & 2,44 & $-2,66$ & $\mathrm{~N}$ & 2,43 & $-2,65$ & $\mathrm{~N}$ \\
\hline 8101 & 3,00 & $-3,18$ & $\mathrm{~N}$ & 2,80 & $-3,21$ & $\mathrm{~N}$ & 2,90 & $-3,22$ & $\mathrm{~N}$ & 2,95 & $-3,24$ & $\mathrm{~N}$ \\
\hline 8112 & 32,42 & $-11,39$ & S & 33,73 & $-11,62$ & $\mathrm{~S}$ & 33,89 & $-11,64$ & $\mathrm{~S}$ & 33,84 & $-11,64$ & $\mathrm{~S}$ \\
\hline 8207 & 8,14 & $-5,61$ & $\mathrm{~S}$ & 8,31 & $-5,64$ & S & 8,20 & $-5,64$ & S & 8,18 & $-5,63$ & $\mathrm{~S}$ \\
\hline 8408 & 2,60 & $-2,57$ & $\mathrm{~N}$ & 2,56 & $-2,60$ & $\mathrm{~N}$ & 2,20 & $-2,52$ & $\mathrm{~N}$ & 4,56 & $-2,63$ & $\mathrm{~N}$ \\
\hline 8409 & 3,58 & $-3,65$ & $\mathrm{~N}$ & 3,26 & $-3,32$ & $\mathrm{~N}$ & 4,52 & $-3,47$ & S & 5,44 & $-3,87$ & $\mathrm{~S}$ \\
\hline 8411 & 7,49 & $-5,42$ & S & 8,41 & $-5,71$ & $\mathrm{~S}$ & 8,41 & $-5,71$ & $\mathrm{~S}$ & 8,11 & $-5,60$ & $\mathrm{~S}$ \\
\hline 8412 & 4,60 & $-3,97$ & $\mathrm{~S}$ & 3,78 & $-4,10$ & $\mathrm{~S}$ & 3,92 & $-3,78$ & $\mathrm{~N}$ & 4,10 & $-3,69$ & $\mathrm{~N}$ \\
\hline 8413 & 3,30 & $-3,10$ & $\mathrm{~N}$ & 3,27 & $-3,04$ & $\mathrm{~N}$ & 3,37 & $-2,92$ & $\mathrm{~N}$ & 3,37 & $-2,91$ & $\mathrm{~N}$ \\
\hline 8414 & 2,80 & $-1,55$ & $\mathrm{~N}$ & 2,73 & $-1,68$ & $\mathrm{~N}$ & 2,87 & $-1,79$ & $\mathrm{~N}$ & 2,76 & $-1,62$ & $\mathrm{~N}$ \\
\hline 8429 & 3,60 & $-10,05$ & S & 3,35 & $-10,10$ & S & 4,12 & $-10,09$ & $\mathrm{~S}$ & 4,62 & $-10,23$ & $\mathrm{~S}$ \\
\hline 8431 & 0,50 & $-1,56$ & $\mathrm{~N}$ & 1,01 & $-1,32$ & $\mathrm{~N}$ & 1,11 & $-1,85$ & $\mathrm{~N}$ & 2,00 & $-2,69$ & $\mathrm{~N}$ \\
\hline 8471 & 37,26 & $-12,09$ & $\mathrm{~S}$ & 34,37 & $-11,75$ & $\mathrm{~S}$ & 33,88 & $-11,70$ & $\mathrm{~S}$ & 34,47 & $-11,81$ & $\mathrm{~S}$ \\
\hline 8474 & 2,45 & $-1,85$ & $\mathrm{~N}$ & 0,80 & $-1,10$ & $\mathrm{~N}$ & 0,93 & $-0,75$ & $\mathrm{~N}$ & 0,80 & $-0,73$ & $\mathrm{~N}$ \\
\hline 8481 & 2,15 & $-2,59$ & $\mathrm{~N}$ & 1,66 & $-2,18$ & $\mathrm{~N}$ & 2,20 & $-2,72$ & $\mathrm{~N}$ & 1,74 & $-2,42$ & $\mathrm{~N}$ \\
\hline 8482 & 2,42 & $-2,59$ & $\mathrm{~N}$ & 1,73 & $-2,22$ & $\mathrm{~N}$ & 1,70 & $-2,35$ & $\mathrm{~N}$ & 3,55 & $-3,58$ & $\mathrm{~N}$ \\
\hline 8483 & 2,89 & $-3,01$ & $\mathrm{~N}$ & 3,60 & $-3,51$ & $\mathrm{~N}$ & 3,25 & $-3,03$ & $\mathrm{~N}$ & 5,24 & $-4,03$ & $\mathrm{~S}$ \\
\hline 8501 & 7,26 & $-4,83$ & $\mathrm{~S}$ & 6,52 & $-5,01$ & $\mathrm{~S}$ & 6,74 & $-5,07$ & $\mathrm{~S}$ & 6,73 & $-4,71$ & $\mathrm{~S}$ \\
\hline 8503 & 1,71 & $-2,50$ & $\mathrm{~N}$ & 1,70 & $-2,35$ & $\mathrm{~N}$ & 2,00 & $-2,50$ & $\mathrm{~N}$ & 1,70 & $-2,48$ & $\mathrm{~N}$ \\
\hline 8504 & 4,70 & $-3,70$ & $\mathrm{~S}$ & 4,59 & $-3,99$ & $\mathrm{~S}$ & 5,64 & $-4,30$ & S & 5,90 & $-4,43$ & $\mathrm{~S}$ \\
\hline 8517 & 5,30 & $-4,48$ & $\mathrm{~S}$ & 9,48 & $-6,02$ & $\mathrm{~S}$ & 9,64 & $-5,52$ & $\mathrm{~S}$ & 8,90 & $-5,53$ & $\mathrm{~S}$ \\
\hline 8544 & 2,76 & $-3,05$ & $\mathrm{~N}$ & 2,83 & $-2,43$ & $\mathrm{~N}$ & 2,63 & $-2,66$ & $\mathrm{~N}$ & 2,65 & $-2,71$ & $\mathrm{~N}$ \\
\hline 8607 & 2,01 & $-2,76$ & $\mathrm{~N}$ & 0,80 & $-1,28$ & $\mathrm{~N}$ & 0,86 & $-1,30$ & $\mathrm{~N}$ & 0,80 & $-1,28$ & $\mathrm{~N}$ \\
\hline
\end{tabular}

(continua) 


\section{TABELA I (CONTINUAÇÃO)}

\begin{tabular}{|c|c|c|c|c|c|c|c|c|c|c|c|c|}
\hline \multirow[b]{2}{*}{ Setor } & \multicolumn{3}{|c|}{ Desvio padrão móvel 6} & \multicolumn{3}{|c|}{$\operatorname{GARCH}(1,1)$} & \multicolumn{3}{|c|}{ TGARCH $(1,1)$} & \multicolumn{3}{|c|}{ EGARCH $(1,1)$} \\
\hline & F test & $\begin{array}{c}\text { ECM } \\
\text { t-1 }\end{array}$ & $\begin{array}{c}\text { Coint } \\
?\end{array}$ & F test & $\begin{array}{c}\text { ECM } \\
t-1\end{array}$ & $\begin{array}{c}\text { Coint } \\
?\end{array}$ & F test & $\begin{array}{c}\text { ECM } \\
\text { t-1 }\end{array}$ & $\begin{array}{c}\text { Coint } \\
?\end{array}$ & F test & $\begin{array}{c}\text { ECM } \\
\text { t-1 }\end{array}$ & $\begin{array}{c}\text { Coint } \\
?\end{array}$ \\
\hline 8708 & 1,24 & $-0,96$ & $\mathrm{~N}$ & 1,20 & $-1,20$ & $\mathrm{~N}$ & 1,78 & $-2,25$ & $\mathrm{~N}$ & 1,62 & $-1,89$ & $\mathrm{~N}$ \\
\hline 8802 & 10,57 & $-6,46$ & $\mathrm{~S}$ & 10,83 & $-6,60$ & $\mathrm{~S}$ & 10,32 & $-6,39$ & $\mathrm{~S}$ & 11,03 & $-6,58$ & S \\
\hline 8803 & 3,00 & $-2,01$ & $\mathrm{~N}$ & 3,04 & $-2,02$ & $\mathrm{~N}$ & 3,07 & $-2,00$ & $\mathrm{~N}$ & 2,95 & $-1,98$ & $\mathrm{~N}$ \\
\hline 9032 & 3,32 & $-3,34$ & $\mathrm{~N}$ & 2,14 & $-3,10$ & $\mathrm{~N}$ & 2,14 & $-3,01$ & $\mathrm{~N}$ & 1,92 & $-2,84$ & $\mathrm{~N}$ \\
\hline 9303 & 2,47 & $-2,57$ & $\mathrm{~N}$ & 1,20 & $-1,90$ & $\mathrm{~N}$ & 1,40 & $-2,11$ & $\mathrm{~N}$ & 1,54 & $-2,10$ & $\mathrm{~N}$ \\
\hline 9403 & 4,17 & $-3,02$ & $\mathrm{~N}$ & 4,06 & $-2,95$ & $\mathrm{~N}$ & 4,08 & $-2,98$ & $\mathrm{~N}$ & 4,28 & $-3,08$ & $\mathrm{~N}$ \\
\hline 9998 & 3,61 & $-0,36$ & $\mathrm{~N}$ & 4,67 & $-0,88$ & $\mathrm{~N}$ & 3,56 & $-0,54$ & $\mathrm{~N}$ & 3,51 & $-0,49$ & $\mathrm{~N}$ \\
\hline Total & - & - & 41 & - & - & 46 & - & - & 44 & - & - & 47 \\
\hline
\end{tabular}

Nota: Valores absolutos da estatística t estão em parênteses. Os valores críticos do teste $\mathrm{F}$ são 4,35 para o limite superior, e 3,23 para o limite inferior. O valor crítico da estatística $t$ para o teste ECM é -3,82 de acordo com Banerjee; Dolado; Mestre (1998), considerando três regressores e cem observações.

Fonte: Elaboração própria. 